\section{RESEARCH ARTICLE 10.1029/2019JB018050 \\ Special Section: \\ Simulation of Remanent, Transient, and Induced FORC Diagrams for Interacting Particles With Uniaxial, Cubic, and Hexagonal Anisotropy}

Magnetism in the Geosciences - Advances and Perspectives

Key Points:

- First-order reversal curve (FORC) diagrams were simulated numerically for an extended set of FORC diagram types.

- Diagnostic features in remanent, transient, and induced FORCs for interacting uniaxial, cubic, and hexagonal anisotropy are predicted.

- Results compare favorably with experimental data and provide a theoretical framework for interpreting these FORC diagram types

Correspondence to:

R. J. Harrison,

rjh40@esc.cam.ac.uk

Citation:

Harrison, R. J., Zhao, X., Hu, P., Sato, T., Heslop, D., Muxworthy, A. R., et al (2019). Simulation of remanent, transient, and induced FORC diagrams for interacting particles with uniaxial, cubic, and hexagonal anisotropy. Journal of Geophysical Research: Solid Earth, 124, 12,404-12,429. https:// doi.org/10.1029/2019JB018050

Received 20 MAY 2019 Accepted 14 NOV 2019 Accepted article online 21 NOV 2019 Published online 14 DEC 2019

\author{
Richard J. Harrison ${ }^{1}$ iD, Xiang Zhao ${ }^{2,3}$ iD, Pengxiang $\mathrm{Hu}^{2,3}$ iD, Tetsuro Sato ${ }^{3}$, David Heslop ${ }^{2,3}$ (D), \\ Adrian R. Muxworthy ${ }^{4}$, Hirokuni Oda ${ }^{3}$ iD, Venkata S. C. Kuppili ${ }^{1}$, and Andrew P. Roberts ${ }^{2,3}$ iD \\ ${ }^{1}$ Department of Earth Sciences, University of Cambridge, Cambridge, UK, ${ }^{2}$ Research School of Earth Sciences, Australian \\ National University, Canberra, Australia, ${ }^{3}$ Research Institute of Geology and Geoinformation, Geological Survey of Japan, \\ National Institute of Advanced Industrial Science and Technology (AIST), Tsukuba, Japan, ${ }^{4}$ Department of Earth Science \\ and Engineering, Imperial College London, South Kensington Campus, London, UK
}

\begin{abstract}
The diagnostic power of first-order reversal curve (FORC) diagrams has recently been enhanced by an extended measurement protocol that yields three additional FORC-like diagrams: the remanent (remFORC), induced (iFORC), and transient (tFORC) diagrams. Here, we present micromagnetic simulations using this extended protocol, including numerical predictions of remFORC, iFORC, and tFORC signatures for particle ensembles relevant to rock magnetism. Simulations are presented for randomly packed single-domain (SD) particles with uniaxial, cubic, and hexagonal anisotropy, and for chains of uniaxial SD particles. Noninteracting particles have zero tFORC, but distinct remFORC and iFORC signals, that provide enhanced discrimination between uniaxial, cubic, and hexagonal anisotropy types. Increasing interactions lessen the ability to discriminate between uniaxial and cubic anisotropy but reproduces a change in the pattern of positive and negative iFORC signals observed for SD-dominated versus vortexdominated samples. Interactions in SD particles lead to the emergence of a bi-lobate tFORC distribution, which is related to formation of flux-closure in super-vortex states. A predicted iFORC signal associated with collapsed chains is observed in experimental data and may aid magnetofossil identification in sediments. Asymmetric FORC and FORC-like distributions for hexagonal anisotropy are explained by the availability of multiple easy axes within the basal plane. A transition to uniaxial switching occurs below a critical value of the out-of-plane/in-plane anisotropy ratio, which may allow FORC diagrams to provide insight into the stress state of hexagonal minerals, such as hematite.
\end{abstract}

\section{Introduction}

First-order reversal curve (FORC) diagrams provide a powerful method to characterize the distribution of domain states in natural samples (Pike et al., 1999; Roberts et al., 2000; Roberts et al., 2014). The advantage of FORCs lies in the two-dimensional nature of the FORC diagram: The horizontal axis provides information related to the coercivity distribution, while the vertical axis provides additional sensitivity to the presence of viscous superparamagnetic (SP), single-domain (SD), single-vortex (SV), multivortex (MV), and multidomain (MD) states. FORC diagrams also provide a way to discriminate between minerals with different anisotropy types and to detect the presence of interparticle magnetostatic interactions. Combined with recently developed methods to quantify FORC diagrams of multicomponent mixtures (Harrison et al., 2018; Lascu et al., 2015; Ludwig et al., 2013), FORC diagrams are an essential part of the rock-magnetic toolkit and help to alleviate some of the ambiguities associated with popular parametric-ratio methods for domain state classification (Day et al., 1977; Roberts et al., 2018; Roberts et al., 2019).

Our ability to interpret FORC diagrams relies heavily on empirical observations of well-characterized samples made over the last 20 years (see Roberts et al., 2014, and references therein for a review of this work). This empirical knowledge is further supported by theory and simulations, much of which is based on robust micromagnetic principles (Carvallo et al., 2003; Chang et al., 2018; Egli, 2006; Egli \& Winklhofer, 2014; Harrison \& Lascu, 2014; Lanci \& Kent, 2018; Lascu et al., 2018; Muxworthy et al., 2004; Newell, 2005; Pike, Roberts, Dekkers et al., 2001; Pike, Roberts, \& Verosub, 2001; Roberts et al., 2017; Valdez-Grijalva et al., 2018; Valdez-Grijalva \& Muxworthy, 2019). Despite this, FORC diagram interpretation is not always 
straightforward or unambiguous. Ambiguity can be caused by overlapping contributions associated with different aspects of the magnetization process (e.g., field-induced switching, thermal relaxation, coherent rotation, domain wall movement, and vortex nucleation and annihilation). To address this issue, a new extended FORC measurement protocol has recently been developed (Zhao et al., 2017), which enables the FORC signal to be expressed as the sum of three separate FORC-like signals: the remanent FORC (remFORC), induced FORC (iFORC), and transient FORC (tFORC) components. The remFORC diagram contains information about irreversible remanent state changes of a sample. Such changes are associated with irreversible magnetization switching between easy axes (for noninteracting particles) or between local energy minimum states (for strongly interacting particles). The iFORC diagram contains information about reversible magnetization changes, such as spin rotation or domain wall bowing. The tFORC diagram contains information about irreversible switching driven purely by self-demagnetizing or interaction fields (as opposed to switching driven by applied magnetic field reversal) and viscous magnetization changes driven by thermal relaxation. Separating the FORC signal into these three components provides additional diagnostic power because some magnetization processes are either dominantly or exclusively partitioned into one or other of the remFORC, iFORC, and tFORC signals, which makes them easier to isolate and quantify. This approach has led to wider recognition of the importance of, for example, vortex states in rocks, sediments, and soils (Hu et al., 2018; Roberts et al., 2017).

Our current understanding of the information contained within remFORC, iFORC, and tFORC signals is largely empirical, and detailed micromagnetic simulations of the expected form of the resulting FORC-like diagrams have yet to be performed. In this paper we seek to address this gap by adapting the FORCulator micromagnetic simulation method of Harrison and Lascu (2014) to include the extended measurement protocol of Zhao et al. (2017), thereby enabling prediction of the form of remFORC, iFORC, and tFORC signals for particle ensembles relevant to rock magnetism. The method is applied to (1) noninteracting and strongly interacting SD particle ensembles with uniaxial or cubic anisotropy (representing, for example, SD magnetite or greigite in dispersed or clustered arrangements); (2) collapsed chains of uniaxial SD particles (representing bacterial magnetofossils); and (3) noninteracting SD particle ensembles with hexagonal basal-plane anisotropy (representing pseudo-hexagonal magnetic minerals, such as hematite or pyrrhotite). A secondary motivation for this study is to contribute to the development of a set of reference FORC, remFORC, iFORC, and tFORC diagrams for known particle ensembles. Such a data set could provide a qualitative framework to aid experimental data interpretation and could assist in training machine-learning algorithms to automatically recognize diagnostic features of FORC diagrams. The use of machine learning for pattern recognition is well-known in many fields of science, medicine, and engineering (Bishop, 2006), but machine learning has not been applied to automated FORC diagram classification. A major limitation in developing such an approach is the lack of a training set of FORC diagrams that can be assigned to a given category of magnetic behavior. Provided that they are sufficiently representative of experimental results, simulated FORC diagrams provide an attractive method to generate such training data because assignment to a given magnetic behavior class can be made unambiguously as the domain state and particle arrangement of magnetic particles are known exactly for simulations.

\section{Methods}

\subsection{The Extended FORC Measurement Protocol}

The extended FORC measurement protocol of Zhao et al. (2017) is illustrated in Figure 1. Numbered reference points are used to illustrate measurement sequences required to obtain FORC, remFORC, and transient-free (tfFORC) data from which the iFORC and tFORC signals are derived. The protocol begins with a standard FORC measurement (Pike et al., 1999; Roberts et al., 2000). A saturating field $\left(B_{\text {sat }}\right)$ is applied (measurement point 1$)$ and is then decreased to a defined reversal field ( $B_{\mathrm{a}}$ ) (e.g., measurement points 2,3 , or 5 ). An FORC is acquired as the measurement field $\left(B_{\mathrm{b}}\right)$ is swept from $B_{\mathrm{a}}$ back to $B_{\text {sat }}$. The process is repeated to obtain FORCs at a number of reversal fields spanning the range $-B_{\text {sat }} \leq B_{\mathrm{a}} \leq$ to $B_{\text {sat }}$ to yield a magnetization surface, $M_{\mathrm{FORC}}\left(B_{\mathrm{a}}, B_{\mathrm{b}}\right)$. The measurement sequence 1-5-6-7 illustrates part of an FORC with reversal field $B_{\mathrm{a}}=B_{\mathrm{y}}$ and measurement fields $B_{\mathrm{b}}=B_{\mathrm{y}}, B_{\mathrm{z}}$, and 0 . The measurement sequence 1-3-4 represents part of a special FORC, termed a "zero FORC" (Fabian, 2003; Yu \& Tauxe, 2005), which has reversal field $B_{\mathrm{a}}=0$. 


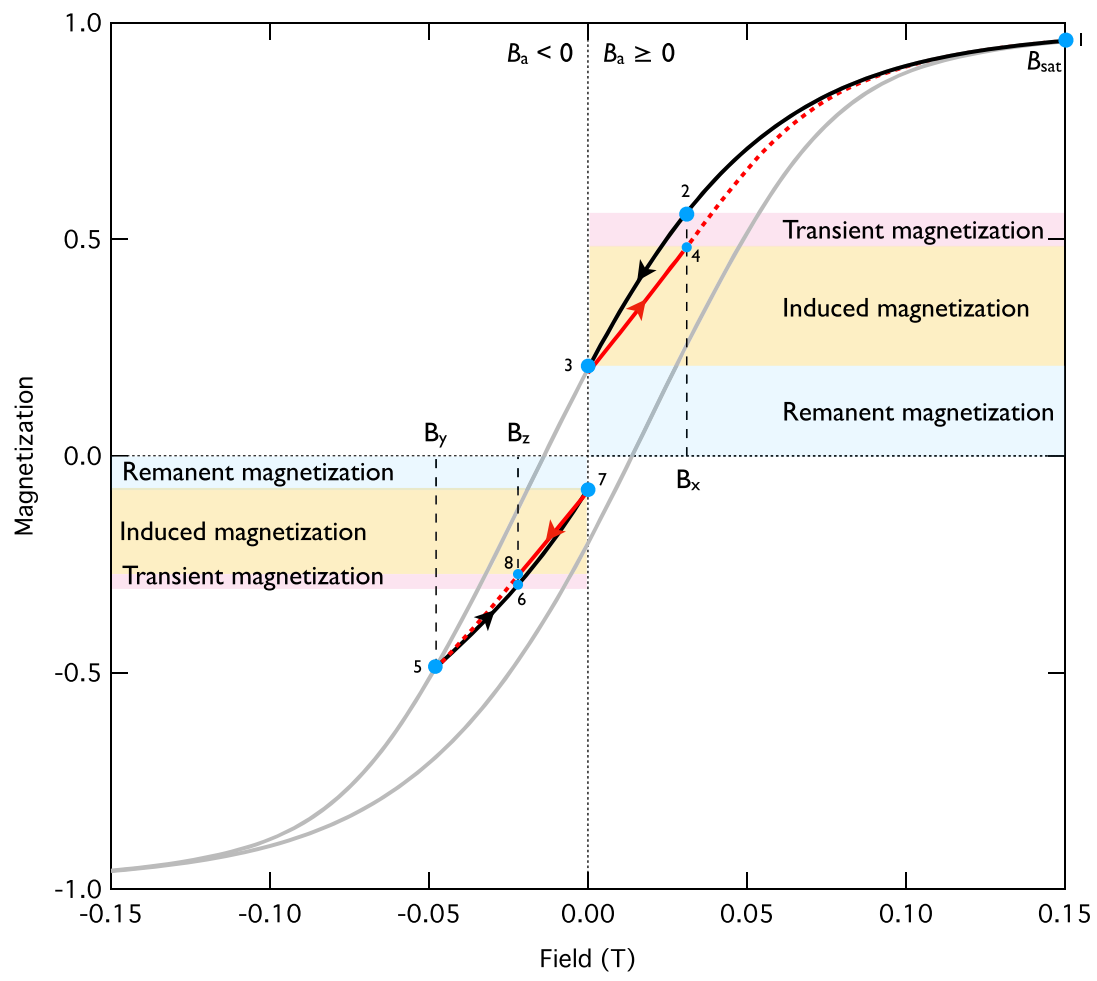

Figure 1. Definition of the remanent, induced, and transient magnetization components and how they are measured using the extended FORC protocol of Zhao et al. (2017). All curves shown are taken from a simulation of randomly packed uniaxial particles with $40 \%$ packing fraction. Measurement point 1 corresponds to the saturating field, $B_{\text {sat }}$, that is applied prior to measurement of each FORC. Measurement points 2, 3, and 5 lie on the descending branch of the hysteresis curve and represent the starting points for conventional first-order reversal curves $M\left(B_{\mathrm{a}}, B_{\mathrm{b}}\right)$ with reversal fields $B_{\mathrm{a}}=B_{\mathrm{x}}, B_{\mathrm{a}}=0$, and $B_{\mathrm{a}}=B_{\mathrm{y}}$, respectively. Measurement point 3 also corresponds to the saturation remanent magnetization, $M_{\mathrm{rs}}$. Measurement sequence 2-3-4 illustrates measurement of the transient-free "zero FORC" curve for positive reversal fields $\left(B_{\mathrm{a}} \geq 0\right)$ : The field is swept from the reversal field $B_{\mathrm{X}}$ to zero (black arrow) and then from zero to $B_{\mathrm{X}}$ (red arrow). Measurement point 4 corresponds to the first point in the transient-free curve, $M_{\mathrm{tf}}\left(B_{\mathrm{x}}, B_{\mathrm{x}}\right)$. Subsequent points, $M_{\mathrm{tf}}$ $\left(B_{\mathrm{X}}, B_{\mathrm{b}}\right)$, are obtained by alternately sweeping the field to zero and then to the next highest measurement field, $B_{\mathrm{b}}$, until saturation. Whenever the field is zero, the corresponding remanent magnetization, $M_{\mathrm{rem}}\left(B_{\mathrm{x}}, B_{\mathrm{b}}\right)$ is recorded.

Measurement sequence 5-7-8 illustrates the measurement of transient-free curves for negative reversal fields $\left(B_{\mathrm{a}}<0\right)$ : The field is swept from the reversal field $B_{y}$ to zero (black arrow) and then from zero to $B_{z}$ (red arrow). Point 6 corresponds to a general point in the conventional FORC curve, $M\left(B_{y}, B_{z}\right)$, and is measured separately in a conventional FORC measurement. Measurement point 8 corresponds to a general point in the transient-free curve, $M_{\mathrm{tf}}\left(B_{\mathrm{y}}, B_{\mathrm{z}}\right)$. The conventional FORC magnetization (e.g. point 6) can be expressed as the sum of three components: a remanent component, $M_{\text {rem }}\left(B_{\mathrm{a}}, B_{\mathrm{b}}\right)$, an induced component, $M_{\mathrm{i}}\left(B_{\mathrm{a}}, B_{\mathrm{b}}\right)=M_{\mathrm{tf}}\left(B_{\mathrm{a}}, B_{\mathrm{b}}\right)-M_{\mathrm{rem}}\left(B_{\mathrm{a}}, B_{\mathrm{b}}\right)$, and a transient component, $M_{\mathrm{t}}\left(B_{\mathrm{a}}, B_{\mathrm{b}}\right)=M\left(B_{\mathrm{a}}, B_{\mathrm{b}}\right)-M_{\mathrm{tf}}\left(B_{\mathrm{a}}, B_{\mathrm{b}}\right)$, indicated by the blue, yellow, and pink shaded regions, respectively.

After a set of standard FORCs has been measured, a second set of measurements is performed to obtain two additional FORC-like data sets: remFORC and tfFORC data. The remFORC is a type of second-order reversal curve (SORC) in which a zero-field measurement point is inserted between each in-field measurement point of the standard FORC protocol (Stancu et al., 2006; Winklhofer et al., 2008). A saturating field is applied to the sample (measurement point 1 ) and is then decreased to zero (measurement point 3 ). The field is then either increased (e.g., measurement point 4) or decreased (e.g., measurement point 5) to the desired reversal field and swept back to zero to obtain the first point in the remFORC, $M_{\mathrm{rem}}\left(B_{\mathrm{a}}, B_{\mathrm{a}}\right)$. For example, measurement sequence 1-3-5-7 yields $M_{\text {rem }}\left(B_{\mathrm{y}}, B_{\mathrm{y}}\right)$. Subsequent points in the remFORC are obtained by alternately sweeping the field from zero to the next measurement field, $B_{a}<B_{\mathrm{b}} \leq B_{\mathrm{sat}}$, and then back to zero. For example, the sequence 7-8-7 would yield $M_{\text {rem }}\left(B_{\mathrm{y}}, B_{\mathrm{z}}\right)$.

The term transient hysteresis was introduced by Fabian (2003) to define the difference in magnetization between a point on the upper branch of the hysteresis loop (e.g., measurement point 2) and the corresponding point on the zero FORC (e.g., measurement point 4). Transient hysteresis is associated with irreversible 
magnetization changes driven by self-demagnetizing fields as the applied field is decreased from positive saturation to zero. By definition, transient hysteresis is zero when the field is zero and remains zero as the field is ramped back in the same direction from which remanence was approached. Hence, magnetization curves that start at a remanence state approached from the positive (negative) field direction, and that are measured as a function of increasing positive (negative) field, are referred to as transient-free curves (Zhao et al., 2017), as shown in red in Figure 1. According to the remFORC protocol described above, every in-field measurement that follows a zero-field measurement corresponds to a point on a transient-free curve (e.g., measurement points 4 and 8). The remFORC protocol inherently contains, therefore, a measurement of the transient-free magnetization, $M_{\mathrm{tf}}\left(B_{\mathrm{a}}, B_{\mathrm{b}}\right)$.

Any point on the FORC magnetization surface, $M_{\mathrm{FORC}}\left(B_{\mathrm{a}}, B_{\mathrm{b}}\right)$, can be described as the sum of three components (Fabian, 2003; Fabian \& von Dobeneck, 1997; Yu \& Tauxe, 2005):

$$
M_{\mathrm{FORC}}\left(B_{a}, B_{b}\right)=M_{\mathrm{rem}}\left(B_{a}, B_{b}\right)+M_{\mathrm{i}}\left(B_{a}, B_{b}\right)+M_{\mathrm{t}}\left(B_{a}, B_{b}\right),
$$

where $M_{\mathrm{i}}\left(B_{\mathrm{a}}, B_{\mathrm{b}}\right)$ is an induced magnetization component and $M_{\mathrm{t}}\left(B_{\mathrm{a}}, B_{\mathrm{b}}\right)$ is the transient hysteresis magnetization. Here, we follow Zhao et al. (2017) by including within the definition of $M_{\mathrm{t}}\left(B_{\mathrm{a}}, B_{\mathrm{b}}\right)$ transient magnetization caused by thermal relaxation effects. From Figure 1, it can be seen that

$$
\begin{gathered}
M_{\mathrm{i}}\left(B_{\mathrm{a}} B_{\mathrm{b}}\right)=M_{\mathrm{tf}}\left(B_{\mathrm{a}} B_{\mathrm{b}}\right)-M_{\mathrm{rem}}\left(B_{\mathrm{a}} B_{\mathrm{b}}\right) \\
M_{\mathrm{t}}\left(B_{\mathrm{a}} B_{\mathrm{b}}\right)=M_{\mathrm{FORC}}\left(B_{\mathrm{a}} B_{\mathrm{b}}\right)-M_{\mathrm{tf}}\left(B_{\mathrm{a}} B_{\mathrm{b}}\right) .
\end{gathered}
$$

From the four magnetization surfaces $\left(M_{\mathrm{FORC}}, M_{\mathrm{rem}}, M_{\mathrm{i}}\right.$, and $\left.M_{\mathrm{t}}\right)$ the corresponding FORC, remFORC, iFORC, and tFORC distributions are defined as

$$
\begin{aligned}
\rho_{\mathrm{FORC}} & =-\frac{1}{2} \frac{\partial^{2} M_{\mathrm{FORC}}}{\partial B_{\mathrm{a}} \partial B_{\mathrm{b}}}, \\
\rho_{\mathrm{rem}} & =-\frac{1}{2} \frac{\partial^{2} M_{\mathrm{rem}}}{\partial B_{\mathrm{a}} \partial B_{\mathrm{b}}}, \\
\rho_{\mathrm{i}} & =-\frac{1}{2} \frac{\partial^{2} M_{\mathrm{i}}}{\partial B_{\mathrm{a}} \partial B_{\mathrm{b}}}
\end{aligned}
$$

and

$$
\rho_{\mathrm{t}}=-\frac{1}{2} \frac{\partial^{2} M_{\mathrm{t}}}{\partial B_{\mathrm{a}} \partial B_{\mathrm{b}}} .
$$

\subsection{FORCulator Micromagnetic Simulations}

FORCulator is a micromagnetic tool for simulating FORC diagrams for ensembles of interacting SD particles (Harrison \& Lascu, 2014). We provide here a brief overview of the method, together with a description of the changes required to incorporate the extended measurement protocol and particles with hexagonal anisotropy. Other details of the theory and method used to perform FORC simulations are unchanged from those described by Harrison and Lascu (2014).

\subsubsection{Overview of the Micromagnetic Method}

Particles are treated as freely rotating point dipoles with a specified magnetic moment and anisotropy type (either uniaxial, cubic, or hexagonal). The magnetization vector for the $i$ th particle is denoted $\boldsymbol{m}_{\boldsymbol{i}}$. Each particle experiences an effective magnetic field, $\boldsymbol{B}_{\boldsymbol{i}}^{e f f}$, which is the sum of the applied field, $\boldsymbol{B}$, an anisotropy field, $\boldsymbol{B}_{\boldsymbol{i}}^{\boldsymbol{a n i}}$, and a magnetostatic interaction field, $\boldsymbol{B}_{\boldsymbol{i}}^{\text {int }}$, generated by all other particles in the ensemble. A local energy minimum (LEM) state of the ensemble occurs when all $\boldsymbol{m}_{\boldsymbol{i}}$ are parallel to their corresponding $\boldsymbol{B}_{\boldsymbol{i}}^{\text {eff }}$. For any given magnetic configuration of the ensemble, $\boldsymbol{m}_{\boldsymbol{i}}$ deviates from $\boldsymbol{B}_{\boldsymbol{i}}^{\text {eff }}$ by an angle $\delta_{i}$ and experiences a corresponding torque, $\tau_{\boldsymbol{i}}=\boldsymbol{m}_{\boldsymbol{i}} \times \boldsymbol{B}_{\boldsymbol{i}}^{\text {eff }}$, that causes the moment to precess around the effective field. Dynamic time integration of the Landau-Lifshitz-Gilbert (LLG) equation provides the most physically meaningful 
pathway to the nearest LEM state, but it is too slow to enable the thousands of field steps required to be calculated on a practical timescale. Instead, energy minimization is sought by rotating $\boldsymbol{m}_{\boldsymbol{i}}$ directly toward $\boldsymbol{B}_{\boldsymbol{i}}^{\text {eff }}$ by an amount $f \delta$, where $0<f<1$ is a damping factor. In favorable cases, $f$ values close to one allow rapid convergence of the system toward an LEM. However, for some anisotropy types, e.g., cubic anisotropy with $<100>$ easy axes and hexagonal anisotropy, large $f$ leads to oscillatory solutions that do not converge. Reducing $f$ in those cases leads to stable solutions, at the expense of increasing the number of iterations needed to achieve convergence. Convergence is achieved when the mean magnitude of the torque is below a certain value:

$$
\tau=\frac{1}{N} \sum_{i=1}^{N}\left|\boldsymbol{m}_{\boldsymbol{i}} \times \boldsymbol{B}_{\boldsymbol{i}}^{e f f}\right|<C_{\text {lim }}
$$

A value of $C_{\lim }=10^{-4}$ was used throughout this paper.

\subsubsection{Incorporation of Hexagonal Anisotropy}

As well as the uniaxial and cubic anisotropy cases explored by Harrison and Lascu (2014), we include here simulations for hexagonal anisotropy within a basal plane, which is relevant for hematite or pyrrhotite. Unit vectors $\boldsymbol{c}_{\boldsymbol{x}}, \boldsymbol{c}_{\boldsymbol{y}}$, and $\boldsymbol{c}_{\boldsymbol{z}}$ represent three orthogonal reference axes, with $\boldsymbol{c}_{\boldsymbol{x}}$ and $\boldsymbol{c}_{\boldsymbol{y}}$ lying within the basal plane and $\boldsymbol{c}_{\boldsymbol{z}}$ lying normal to the basal plane. A uniaxial out-of-plane (oop) anisotropy energy is defined as

$$
E_{\text {oop }}=K_{U} \sin ^{2}(\theta)
$$

where $\theta$ is the angle between $\boldsymbol{m}_{\boldsymbol{i}}$ and $\boldsymbol{c}_{\boldsymbol{z}}$. For large negative $K_{\mathrm{u}}$ values, energy is minimized when $\theta=90^{\circ}$, which forces moments to remain close to the $\boldsymbol{c}_{\boldsymbol{x}}-\boldsymbol{c}_{\boldsymbol{y}}$ plane. Within that plane, moments are exposed to a hexagonal in-plane (ip) anisotropy energy of the form:

$$
E_{i p}=K_{H} \cos (6 \phi)
$$

where $\phi$ is the azimuthal angle between $\boldsymbol{m}_{\boldsymbol{i}}$ and $\boldsymbol{c}_{\boldsymbol{x}}$. Equation (10) describes anisotropy with sixfold symmetry (six easy and six hard directions). Corresponding contributions to the effective field are

$$
\begin{gathered}
\boldsymbol{B}_{\mathbf{U}}^{\mathrm{ani}}=\frac{2 K_{U}}{M_{s}} m_{z} \boldsymbol{c}_{\mathbf{z}}, \text { and } \\
\boldsymbol{B}_{\mathbf{H}}^{\mathbf{a n i}}=-\frac{2 K_{H}}{M_{s}}\left(6 m_{x}^{5}-60 m_{x}^{3} m_{y}^{2}+30 m_{x} m_{y}^{4}\right) \boldsymbol{c}_{\mathbf{x}}-\frac{2 K_{H}}{M_{s}}\left(-30 m_{x}^{4} m_{y}+60 m_{x}^{2} m_{y}^{3}-6 m_{y}^{5}\right) \boldsymbol{c}_{\mathbf{y}},
\end{gathered}
$$

where $M_{\mathrm{s}}$ is the saturation magnetization. For convenience, the anisotropy constants are specified using switching-field parameters $B_{\mathrm{U}}=2 K_{\mathrm{U}} / M_{\mathrm{S}}$ and $B_{\mathrm{H}}=2 K_{\mathrm{H}} / M_{\mathrm{s}}$. For positive $B_{\mathrm{H}}$, the easy axes lie at $30^{\circ}, 90^{\circ}$, $150^{\circ}, 210^{\circ}, 270^{\circ}$, and $330^{\circ}$ from $\boldsymbol{c}_{\boldsymbol{x}}$, with hard axes at $0^{\circ}, 60^{\circ}, 120^{\circ}, 180^{\circ}, 240^{\circ}$, and $300^{\circ}$. Small damping factors of $f=0.02-0.06$, with maximum iterations set to 1500 , were used to obtain stable solutions for hexagonal simulations.

\subsubsection{Incorporating the Extended Measurement Protocol Into FORCulator}

The first stage of our simulations uses the method of Harrison and Lascu (2014) to simulate a conventional FORC diagram. Simulations were first performed as a function of field from $B_{\text {sat }}$ to $-B_{\text {sat }}$, to define the upper branch of the hysteresis loop and to store the magnetic configuration of the ensemble at each reversal field, $B_{\mathrm{a}}$. These stored configurations are used for the remFORC simulations, as discussed below. Simulations are then performed for each FORC by sweeping the field from $B_{\mathrm{a}}$ to $B_{\text {sat }}$. For FORCs with $B_{\mathrm{a}} \leq 0$ (e.g., measurement sequence 5-6-7 in Figure 1), the measurement field, $B_{\mathrm{b}}$, passes through zero, which allows a record to be kept of the magnetic configuration of the ensemble in each back-field remanent state (e.g., measurement point 7 in Figure 1). These stored configurations are also used for the remFORC simulations, as discussed below. For remFORC simulations, the protocols used for $B_{\mathrm{a}} \geq 0$ and $B_{\mathrm{a}}<0$ differ slightly. For $B_{\mathrm{a}} \geq 0$, initialization of each remFORC simulation is always the same and corresponds to the stored magnetic configuration of the saturation remanent state (measurement point 3 in Figure 1). The simulation then proceeds by alternately setting the field to the desired measurement field (tfFORC) and then back to zero (remFORC). For $B_{\mathrm{a}}<0$, there are two options for initializing the simulation. In Option 1, the simulation is initialized using the stored magnetic configuration of the ensemble at the reversal field (e.g., measurement point 5 in 
Figure 1). The simulation then proceeds by alternately setting the field to zero (remFORC) and then to the desired measurement field (tfFORC). In Option 2, the simulation is initialized with the stored magnetic configuration of the back-field remanence state (e.g., point 7 in Figure 1). The simulation then proceeds by alternately setting the field to the desired measurement field (tfFORC) and then back to zero (remFORC).

Note that the remFORC simulation protocol makes repeated large applied field increments and decrements. The LEM state obtained after a single large field increment or decrement may differ significantly from that obtained by gradually stepping the field to the same value. Although small field steps are always desirable in micromagnetic simulations, it would be prohibitively expensive computationally to sweep the measurement field and back again repeatedly. Possible errors and artefacts related to the use of large field increments are considered in section 4.2 .

The extended measurement protocol of Zhao et al. (2017) employs a variable resolution grid of reversal and measurement fields. An irregular grid provides more efficient sampling of the FORC space, by focusing more measurements in regions where the magnetization changes most rapidly (Zhao et al., 2015). The extended protocol does not require the use of irregular grids; we here adopt a regular grid with constant field step sizes used for both reversal fields and measurement fields. Choice of a regular grid is driven primarily by the desire to use consistent sampling for all simulations. As long as the FORC space is sufficiently well sampled in both cases, the choice of irregular versus regular grids has no impact on comparisons of measured versus simulated data.

\subsubsection{Simulation and Smoothing Parameters Used}

All simulations were performed using 151 FORCs, $B_{\text {sat }}=0.15 \mathrm{~T}$, and a uniform field-step size of $2 \mathrm{mT}$ for both $B_{\mathrm{a}}$ and $B_{\mathrm{b}}$. Particles were assigned a magnetic moment equivalent to that of a magnetite sphere with $100 \mathrm{~nm}$ diameter. Interacting clusters of 50-500 randomly oriented particles were created using either random (volume packing fractions 0 to $40 \%$ ) or face-centered-cubic (fcc) arrangements. For fcc arrangements, center-center separations of 110 and $100 \mathrm{~nm}$ were used, which correspond to packing fractions of 56\% and $74 \%$, respectively. Chain configurations were created using the constrained, self-avoiding random walk procedure of Harrison and Lascu (2014). Chain collapse is defined by the chain collapse factor $0<c<1$, where $c=0$ corresponds to perfectly straight chains and $c=1$ corresponds to the most collapsed chain. Particle separations within chains were $110 \mathrm{~nm}$, and uniaxial anisotropy axes were aligned with the chain axis. Chains were oriented randomly and do not interact magnetically with each other. For uniaxial anisotropy simulations, particles were assigned random switching fields with log-normal distribution:

$$
\rho\left(B_{\mathrm{U}}\right)=\frac{1}{\sigma \sqrt{2 \pi}} \exp \left[-\frac{1}{2}\left[\frac{\ln \left[\beta B_{\mathrm{U}}\right]}{\sigma}\right]^{2}\right],
$$

with $\sigma=0.5$ and $\beta=20$ (Harrison \& Lascu, 2014). These parameters yield a coercivity distribution typical of those encountered in magnetite-bearing rocks. For cubic anisotropy simulations, switching-field parameters $B_{\mathrm{C}}=3 B_{\mathrm{U}}$ were used, so that resulting coercivity distributions are roughly comparable to uniaxial simulations. For simulations with hexagonal anisotropy, all particles were assigned identical switching fields. Simulations with a range of $B_{\mathrm{U}} / B_{\mathrm{H}}$ ratios were performed. In each case, absolute $B_{\mathrm{U}}$ and $B_{\mathrm{H}}$ values were adjusted to yield roughly comparable coercivities to the uniaxial and cubic cases. Results represent the average of 100-200 simulations. FORC, remFORC, iFORC, and tFORC distributions (equations (4)-(7)) were processed in FORCinel (Harrison \& Feinberg, 2008) using VARIFORC smoothing (Egli, 2013). Processed diagrams are presented using $B_{\mathrm{a}}, B_{\mathrm{b}}$ axes, rather than the more usual $B_{\mathrm{c}}=\left(B_{\mathrm{b}}-B_{\mathrm{a}}\right) / 2, B_{\mathrm{u}}=\left(B_{\mathrm{b}}+B_{\mathrm{a}}\right) / 2$ axes, to facilitate direct comparison of simulations with data presented by Zhao et al. (2017), Roberts et al. (2017), and Hu et al. (2018).

\section{Results}

Simulated FORCs, remFORCs, iFORCs, and tFORCs for noninteracting uniaxial, interacting uniaxial (packing fraction 40\%), noninteracting cubic, interacting cubic (packing fraction 40\%), straight uniaxial chains, and noninteracting hexagonal particles are shown in Figures 2-5. Summaries of the evolution in processed FORC, remFORC, iFORC, and tFORC diagrams as a function of either packing fraction, chain collapse, or $B_{\mathrm{U}} / B_{\mathrm{H}}$ ratio, are shown in Figures 6-9. Key features of individual cases are highlighted below. Except for 

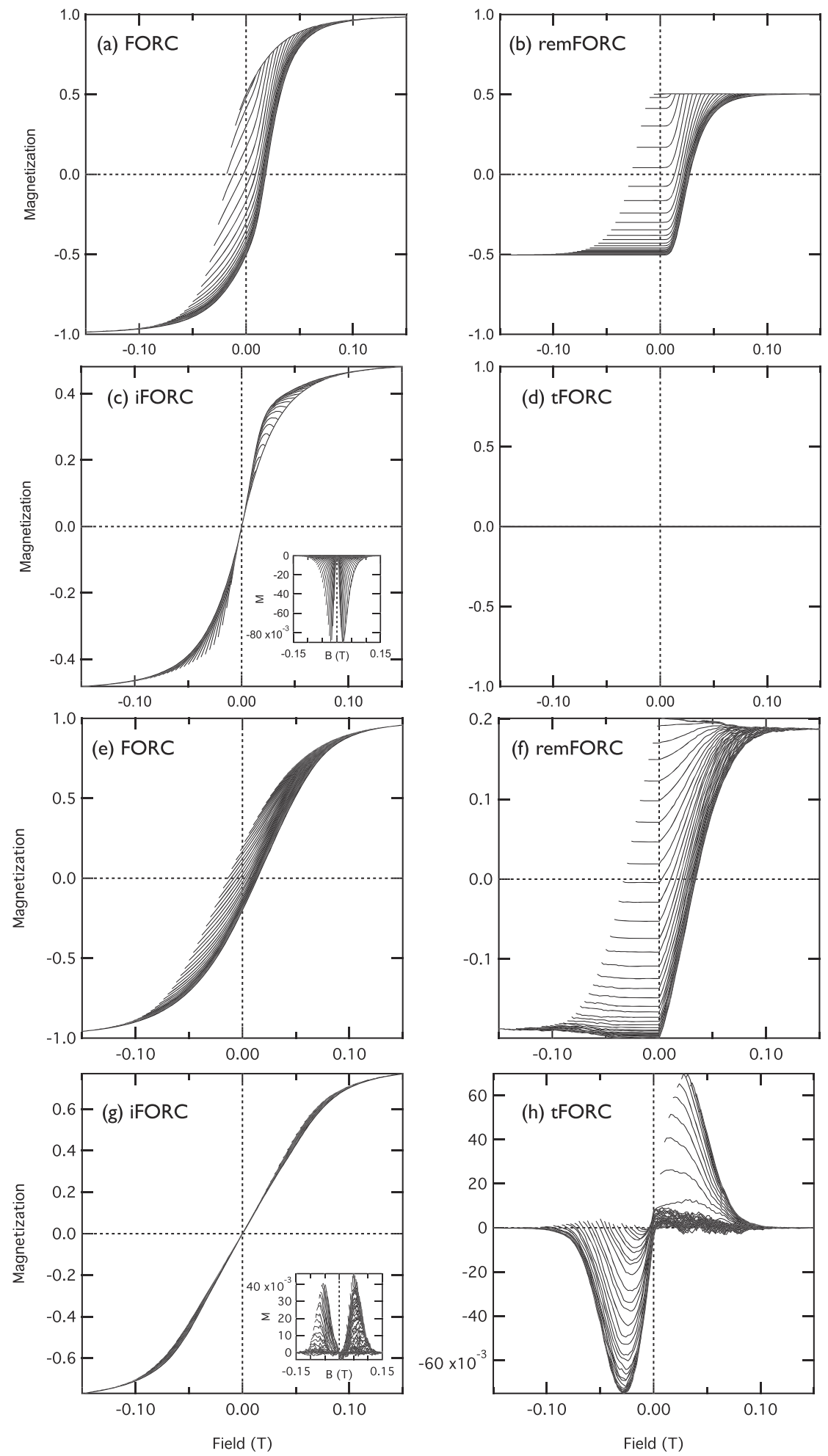

Figure 2. Simulated (a) FORC, (b) remFORC, (c) iFORC, and (d) tFORC curves for randomly oriented, non-interacting particles with uniaxial anisotropy. All simulations consist of 151 curves with a uniform $2 \mathrm{mT}$ step size for both $B_{\mathrm{a}}$ and $B_{\mathrm{b}}$. For clarity, every fourth curve is shown. Magnetization values are normalized to $M_{\mathrm{S}}=1$. Results are the average of 20,000 particles. Simulated (e) FORC, (f) remFORC, (g) iFORC, and (h) tFORC curves for randomly oriented particles with uniaxial anisotropy and packing fraction of $40 \%$. All simulations consist of 151 curves with a uniform $2 \mathrm{mT}$ step size for both $B_{\mathrm{a}}$ and $B_{\mathrm{b}}$. For clarity, every second curve is shown. Magnetization values are normalized relative to $M_{\mathrm{S}}=1$. Each simulation contained 500 particles. Results are the average of 100 simulations ( 50,000 particles). 

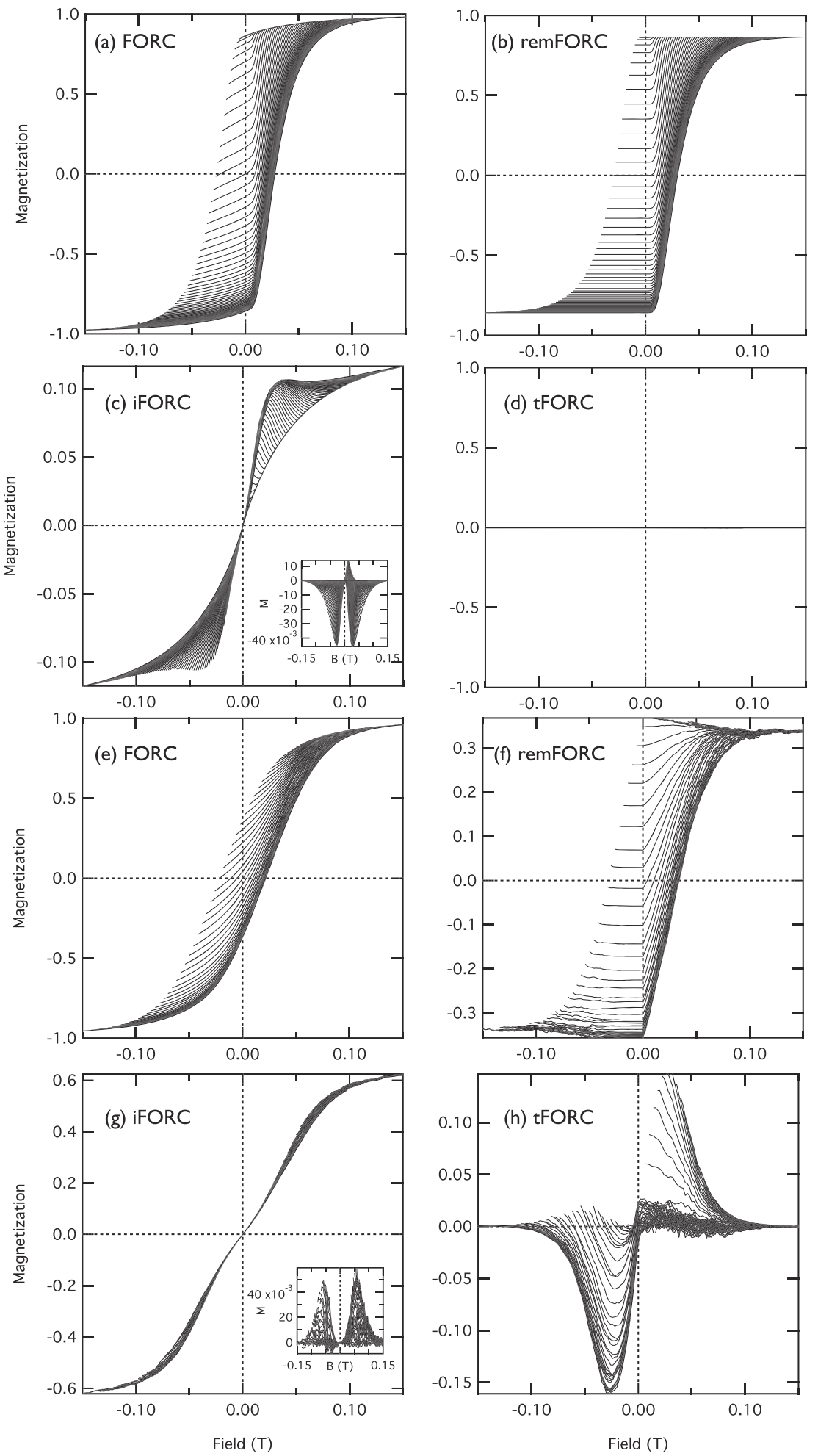

Figure 3. Simulated (a) FORC, (b) remFORC, (c) iFORC, and (d) tFORC curves for randomly oriented, noninteracting particles with cubic anisotropy. All simulations consist of 151 curves with a uniform $2 \mathrm{mT}$ step size for both $B_{\mathrm{a}}$ and $B_{\mathrm{b}}$. Magnetization values are normalized to $M_{\mathrm{s}}=1$. Results shown are the average of 40,000 particles. Simulated (e) FORC, (f) remFORC, (g) iFORC, and (h) tFORC curves for randomly oriented particles with cubic anisotropy and packing fraction of $40 \%$. All simulations consist of 151 curves with a uniform $2 \mathrm{mT}$ step size for both $B_{\mathrm{a}}$ and $B_{\mathrm{b}}$. For clarity, every second curve is shown. Magnetization values are normalized to $M_{\mathrm{S}}=1$. Each simulation contained 100 particles. Results are the average of 100 simulations (10,000 particles). 

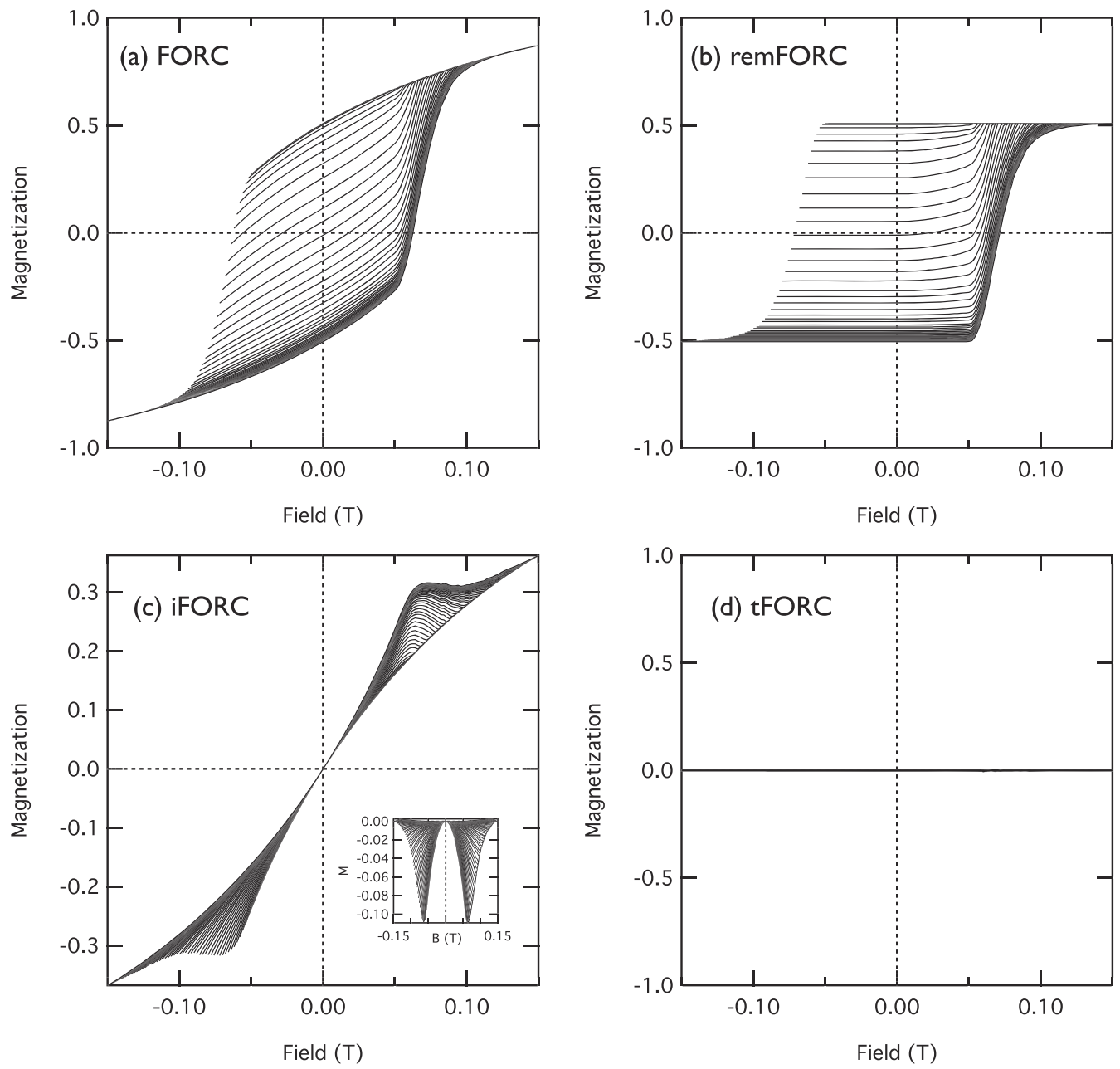

Figure 4. Simulated (a) FORC, (b) remFORC, (c) iFORC, and (d) tFORC curves for randomly oriented, straight chains of uniaxial particles. Chains contained 20 particles with diameter $100 \mathrm{~nm}$ and center-to-center separation of $110 \mathrm{~nm}$. All simulations consist of 151 curves with a uniform $2 \mathrm{mT}$ step size for both $B_{\mathrm{a}}$ and $B_{\mathrm{b}}$. For clarity, every second curve is shown. Magnetization values are normalized to $M_{\mathrm{S}}=1$. Each simulation contained 20 chains. Results are the average of 100 simulations (2000 chains, 40,000 particles).

uniaxial chains, simulations were performed using Option 2 of the remFORC protocol described in section 2.2.3.

\subsection{Results for Randomly-Oriented Uniaxial Particles}

\subsubsection{Conventional FORCs}

Processed FORC diagrams for a range of packing fractions are summarized in Figure 6. Interactions cause obvious changes in the simulated FORC diagrams for packing fractions $\geq 1 \%$. Interactions dominate FORC diagrams for higher packing fractions, representing particles that are clustered more strongly. The most extreme packing fraction used here (74\%) represents a close-packed arrangement of spherical particles in contact, such as that found in framboids. Standard FORC diagrams (Figures 6a-6d) reproduce the results of Harrison and Lascu (2014), with the well-established evolution from central ridge (Egli et al., 2010; Newell, 2005) to teardrop (Egli, 2006; Pike et al., 1999) to wishbone (Pike et al., 2005), to winged (Pike, Roberts, Dekkers et al., 2001) structures with increasing packing. A prominent negative feature along the negative $B_{\mathrm{u}}$ axis is visible in all cases.

\subsection{2. remFORCs}

Comparison of noninteracting (Figure 2b) and interacting (Figure 2f) SD particles reveals several anomalous features in raw remFORCs for the interacting case. We observe $M_{\mathrm{rem}}\left(B_{\mathrm{a}}<0, B_{\mathrm{a}} \leq B_{\mathrm{b}} \leq 0\right)=$ constant $=M_{\text {rem }}$ 

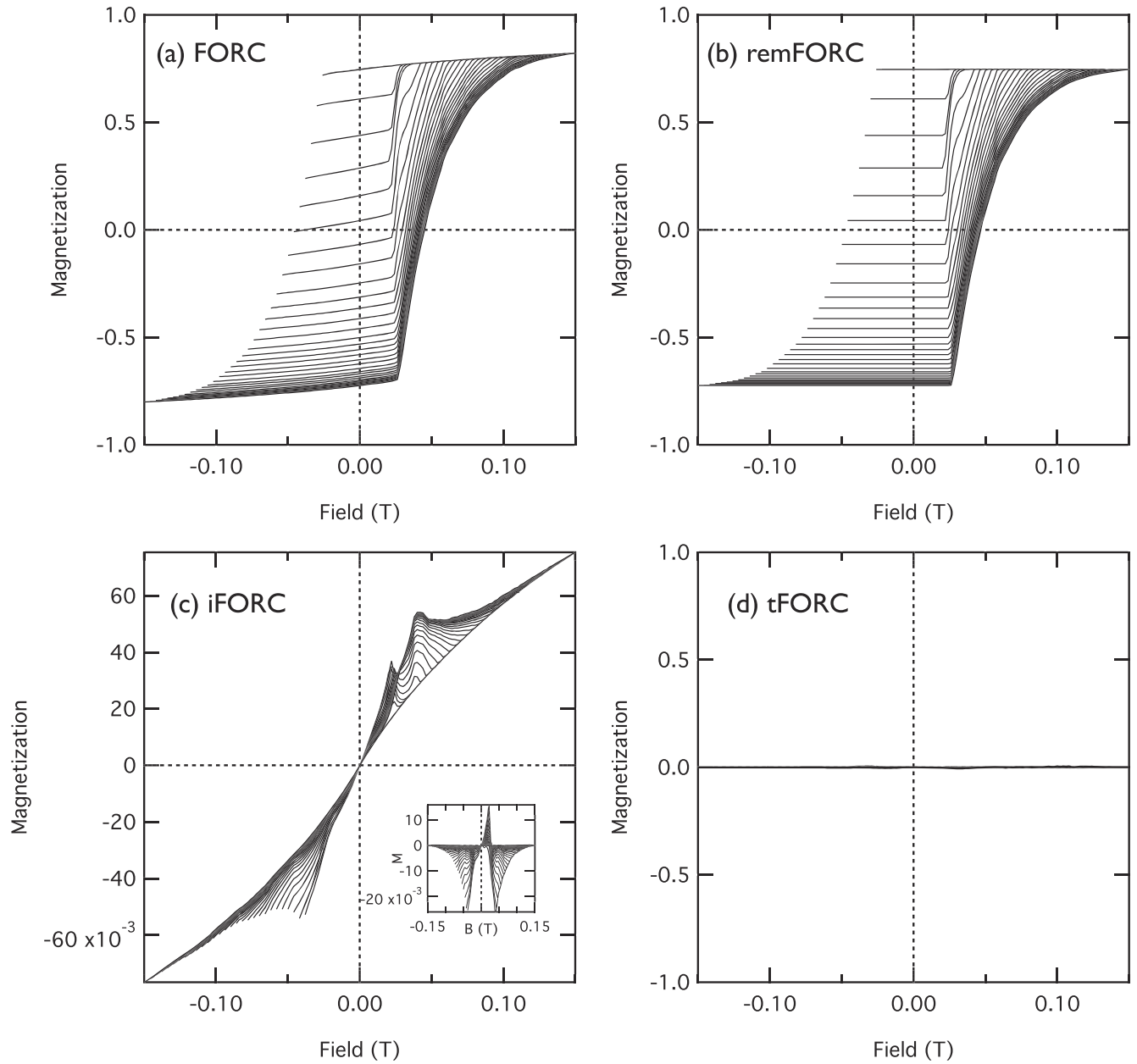

Figure 5. Simulated (a) FORC, (b) remFORC, (c) iFORC, and (d) tFORC curves for randomly oriented, noninteracting particles with hexagonal anisotropy and $K_{\mathrm{u}} / K_{\mathrm{h}}=-333$. All simulations consist of 151 curves with a uniform $2 \mathrm{mT}$ step size for both $B_{\mathrm{a}}$ and $B_{\mathrm{b}}$. For clarity, every second curve is shown. Magnetization values are normalized to $M_{\mathrm{s}}=1$. Simulations contained 50 particles. Results are the average of 100 simulations (5000 particles).

$\left(B_{\mathrm{a}}<0, B_{\mathrm{a}}\right)$ in the noninteracting case (Figure 2b), while an initial decrease in $M_{\mathrm{rem}}\left(B_{\mathrm{a}}<0, B_{\mathrm{a}} \leq B_{\mathrm{b}} \leq 0\right)$ with increasing measurement field is observed in the strongly interacting case (Figure 2f). Processed remFORC diagrams (Figures 6e-6h) have almost exclusively positive distributions that are restricted almost entirely to the remanence region of the FORC space, which is bounded by the lines $B_{\mathrm{a}}<0$ and $B_{\mathrm{b}}>0$ (horizontal and vertical dotted lines, respectively, in Figures 6e-6h). The remFORC distribution begins as a positive central ridge for noninteracting particles (Figure 6e), which broadens with increasing interactions until it reaches the bounds of the remanence region (Figure $6 \mathrm{f}$ ). Thereafter, no further broadening is possible, and there is, instead, a gradual "squaring" of the remFORC distribution (Figure $6 \mathrm{~g}$ ) and a reduction in its maximum intensity (Figure 6h) with increasing packing fraction. A weak positive signal along the $-B_{\mathrm{u}}$ axis (outside the remanence region) appears for strongly interacting particles (labelled P* in Figure 6h).

\subsection{3. iFORCs}

For noninteracting particles, $M_{\mathrm{i}}\left(B_{\mathrm{a}}<0, B_{\mathrm{b}}\right)$ typically reaches a maximum in positive measurement fields, followed by a discontinuous change in slope at $B_{\mathrm{b}}=-B_{\mathrm{a}}$ (Figure 2c). Lower-branch-subtracted iFORCs have two negative peaks (Figure 2c inset). For strongly interacting particles, the discontinuous change in slope at $B_{\mathrm{b}}=-B_{\mathrm{a}}$ disappears, and a clear difference in slope at the beginning of each iFORC appears for positive reversal fields (Figure 2g). Lower-branch-subtracted iFORCs have two positive peaks (Figure 2g inset). Processed iFORC diagrams (Figures 6i-61) have a mixture of positive $(\mathrm{P})$ and negative $(\mathrm{N})$ signals in distinct patterns that evolve with increasing packing fraction. For noninteracting particles (Figure 6i), a clear N-P-N 

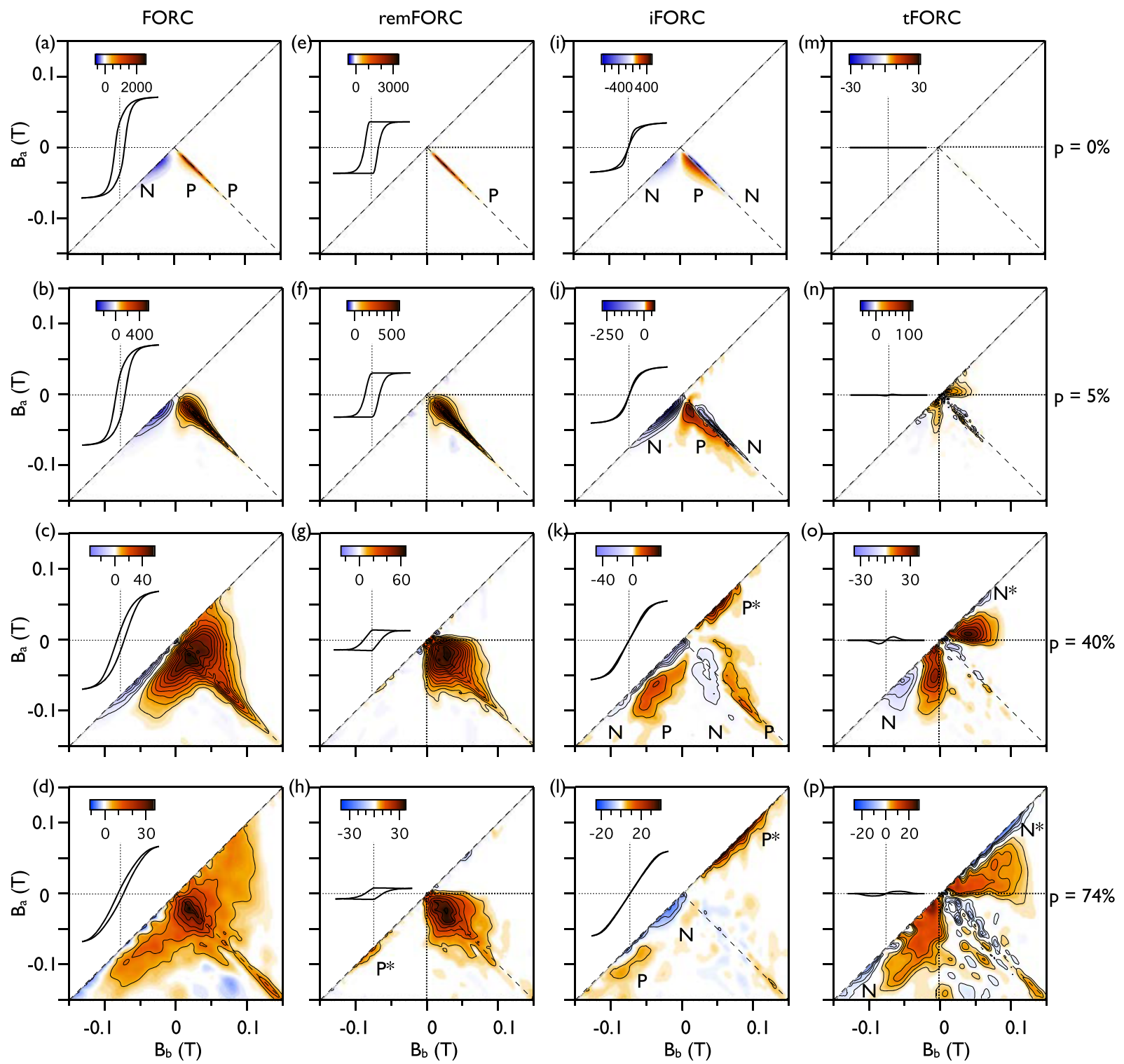

Figure 6. Processed (a-d) FORC, (e-h) remFORC, (i-l) iFORC, and (m-p) tFORC diagrams for randomly packed uniaxial particles with packing fractions (a, e, $\mathrm{i}, \mathrm{m}) 0 \%,(\mathrm{~b}, \mathrm{f}, \mathrm{j}, \mathrm{n}) 5 \%,(\mathrm{c}, \mathrm{g}, \mathrm{k}, \mathrm{o}) 40 \%$, and $(\mathrm{d}, \mathrm{h}, \mathrm{l}, \mathrm{p}) 74 \%$. To achieve $74 \%$ packing efficiency, particles were arranged in a face centered cubic array of hard spheres in contact. Insets are simulated hysteresis loops. Axis range for all insets is $M= \pm 1$ (vertical) and $B= \pm 0.15 \mathrm{~T}$ (horizontal). Magnetization values are normalized to $M_{\mathrm{S}}=1$. Labels $\mathrm{P}$ and $\mathrm{N}$ highlight positive and negative regions of interest. Labels $\mathrm{P}^{*}$ and $\mathrm{N}^{*}$ highlight positive and negative regions that may be simulation artefacts.

signature is evident, which comprises negative and positive background signals and a strong negative central ridge. For 5\% packing (Figure 6j), this N-P-N pattern is still visible, albeit with some disruption to the positive background signal and broadening of the negative central ridge. For $40 \%$ packing (Figure 6k), a change to an N-P-N-P pattern is observed. The negative central ridge is no longer visible, and an additional positive signal (labelled P* in Figure $6 \mathrm{k}$ ) appears along the positive $B_{\mathrm{u}}$ axis. The change from the N-P-N to N-P-N-P pattern coincides with the change from negative to positive lower-branch-subtracted iFORCs (Figuress 2c, $2 \mathrm{~g}$ insets), with the crossover at $\sim 10 \%$ packing. For $74 \%$ packing (Figure 61 ), the iFORC features become weak and poorly resolved within simulation noise. The strongest feature remains the positive signal $\left(\mathrm{P}^{*}\right)$ along the positive $B_{\mathrm{u}}$ axis. 

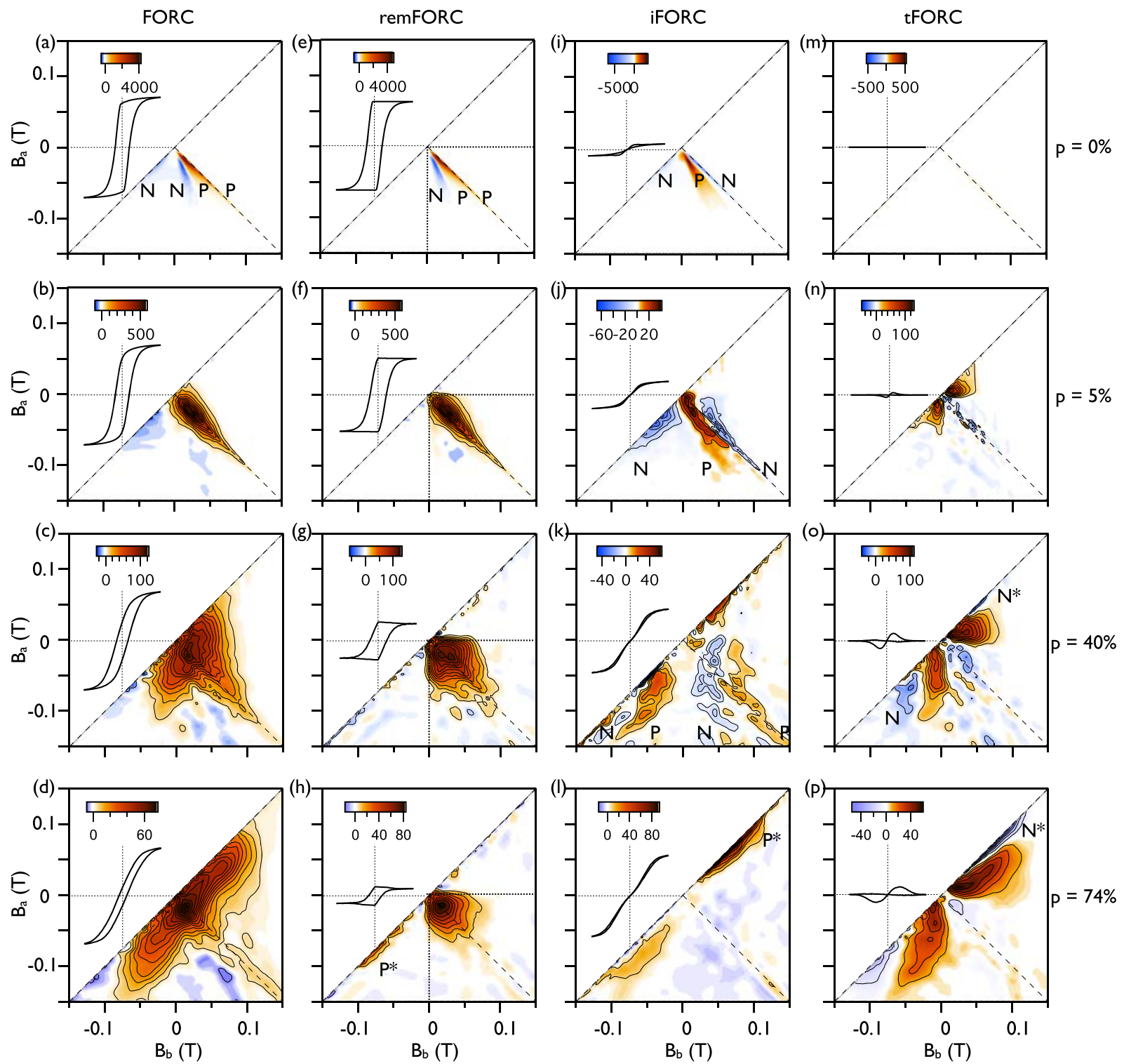

Figure 7. Processed (a-d) FORC, (e-h) remFORC, (i-l) iFORC, and (m-p) tFORC diagrams for randomly packed cubic particles with packing fractions (a, e, i m) $0 \%,(b, f, j, n) 5 \%,(c, g, k, o) 40 \%$, and $(\mathrm{d}, \mathrm{h}, 1, \mathrm{p}) 74 \%$. To achieve $74 \%$ packing efficiency, particles were arranged in a face centered cubic array of hard spheres in contact. Insets are simulated hysteresis loops. Axis range for all insets is $M= \pm 1$ (vertical) and $B= \pm 0.15 \mathrm{~T}$ (horizontal). Magnetization values are normalized to $M_{\mathrm{S}}=1$. Labels $\mathrm{P}$ and $\mathrm{N}$ highlight positive and negative regions of interest. Labels $\mathrm{P}^{*}$ and $\mathrm{N}^{*}$ highlight positive and negative regions that are thought to be simulation artefacts.

\subsection{4. tFORCs}

Noninteracting particles have zero tFORC signal (Figure 2d). Interacting particles develop a double-peaked tFORC structure, with positive and negative peaks observed in positive and negative measurement fields, respectively (Figure $2 \mathrm{~h}$ ). A clear difference in slope at the beginning of each tFORC appears for positive reversal fields (Figure $2 \mathrm{~h}$ ). Processed tFORC diagrams (Figures $6 \mathrm{~m}-6 \mathrm{p}$ ) for interacting particles have a distinct positive bilobate pattern that occupies the two transient regions that bound the remanence region. The two lobes emerge gradually from the origin with increasing packing fraction. For $40 \%$ packing, each lobe forms a distinct peak with closed, closely spaced contours (Figure 60). A distinct negative region (labelled N in Figure 6o) is visible along the negative $B_{\mathrm{u}}$ axis, and a weak negative region (labelled $\mathrm{N}^{*}$ in Figure 60) 

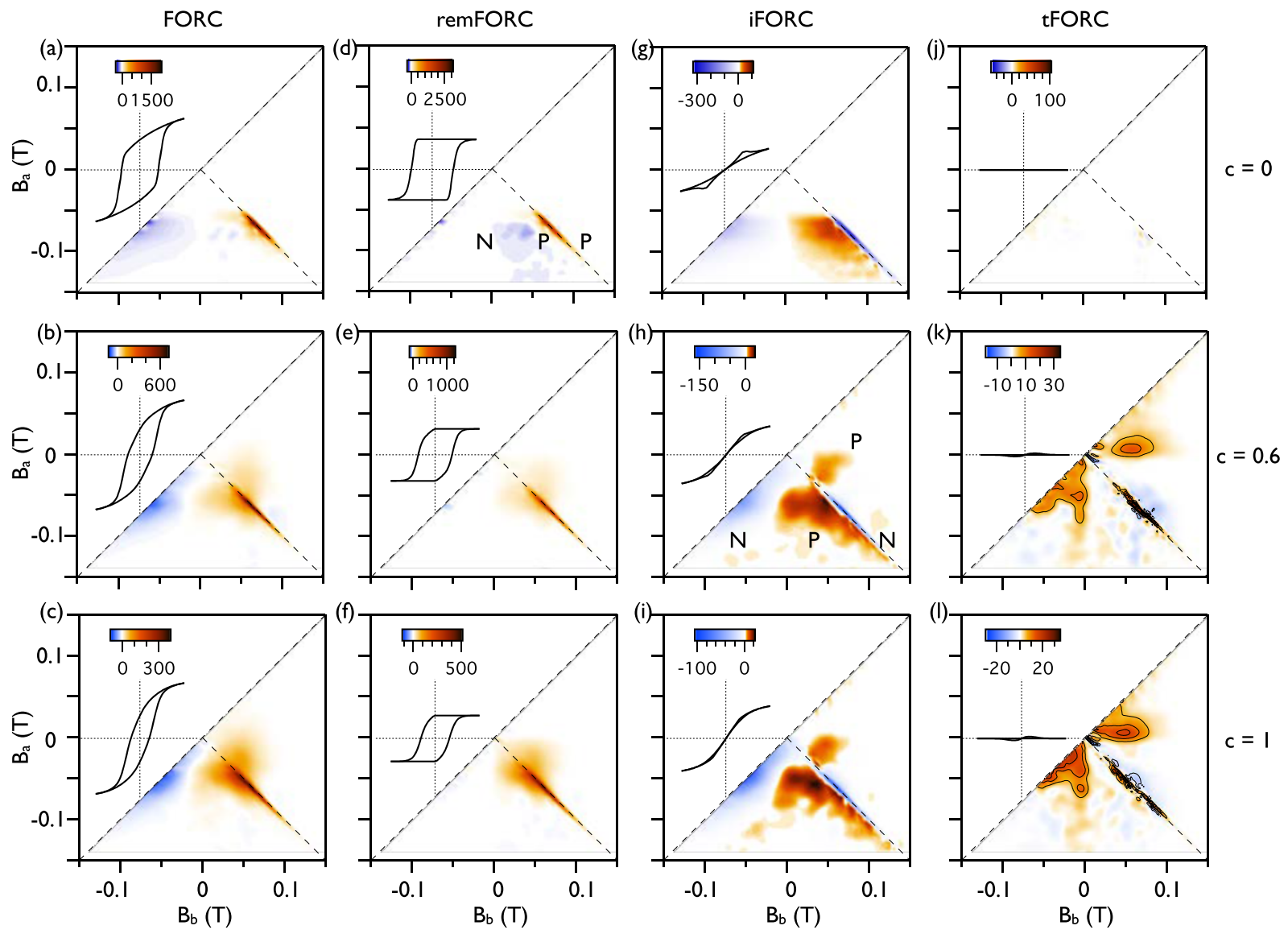

Figure 8. Processed (a-d) FORC, (e-h) remFORC, (i-l) iFORC, and (m-p) tFORC diagrams for chains of uniaxial particles with chain collapse factors (a, d, g, j) $c=0$, (b, e, h, k) $c=0.6$, and (c, f, i, l) $c=1$. Insets are simulated hysteresis loops. Axis range for all insets is $M= \pm 1$ (vertical) and $B= \pm 0.15 \mathrm{~T}$ (horizontal). Magnetization values are normalized relative to $M_{\mathrm{S}}=1$. Labels $\mathrm{P}$ and $\mathrm{N}$ highlight positive and negative regions of interest.

emerges along the positive $B_{\mathrm{u}}$ axis, which mirrors the positive signal $\mathrm{P}^{*}$ in the corresponding iFORC (Figure $6 \mathrm{k}$ ). For $74 \%$ packing, the lobes are spread more broadly with less well-defined peaks and more broadly spaced contours (Figure 6p).

\subsection{Results for Randomly-Oriented Cubic Particles}

\subsubsection{Conventional FORCs}

Processed FORC diagrams for a range of packing fractions are summarized in Figure 7. A FORC diagram for noninteracting particles (Figure 7a) has an N-N-P-P structure, with two negative background signals, a positive background signal, and a positive central ridge. An evolution is observed to teardrop (Figure 7b), wishbone (Figure 7c), and winged (Figure 7d) structures with increasing packing fraction, similar to the uniaxial case. However, both the shape and distribution of the "wings" for $74 \%$ packing are distinct for the uniaxial (Figure 6d) and cubic (Figure 7d) cases.

\subsection{2. remFORCs}

Interacting cubic particles have a pronounced $M_{\mathrm{rem}}\left(B_{\mathrm{a}}>0, B_{\mathrm{b}}\right)$ decrease with increasing measurement field up to $\sim 0.1 \mathrm{~T}$, reaching a value that is considerably lower than $M_{\mathrm{rs}}$ even at the maximum field of $0.15 \mathrm{~T}$ (Figure 3f). An initial decrease in $M_{\mathrm{rem}}\left(B_{\mathrm{a}}<0, B_{\mathrm{a}} \leq B_{\mathrm{b}} \leq 0\right)$ with increasing measurement field is also observed in the strongly interacting case (Figure 3f). In contrast to the uniaxial case, the remFORC diagram for noninteracting cubic particles has an N-P-P structure, with a background negative signal, a positive background signal, and a positive central ridge (Figure 7e). With increasing packing fraction, the negative background signal is quickly swamped by broadening positive background and ridge signals (Figure 7f), and the 
Non-interacting hexagonal anisotropy
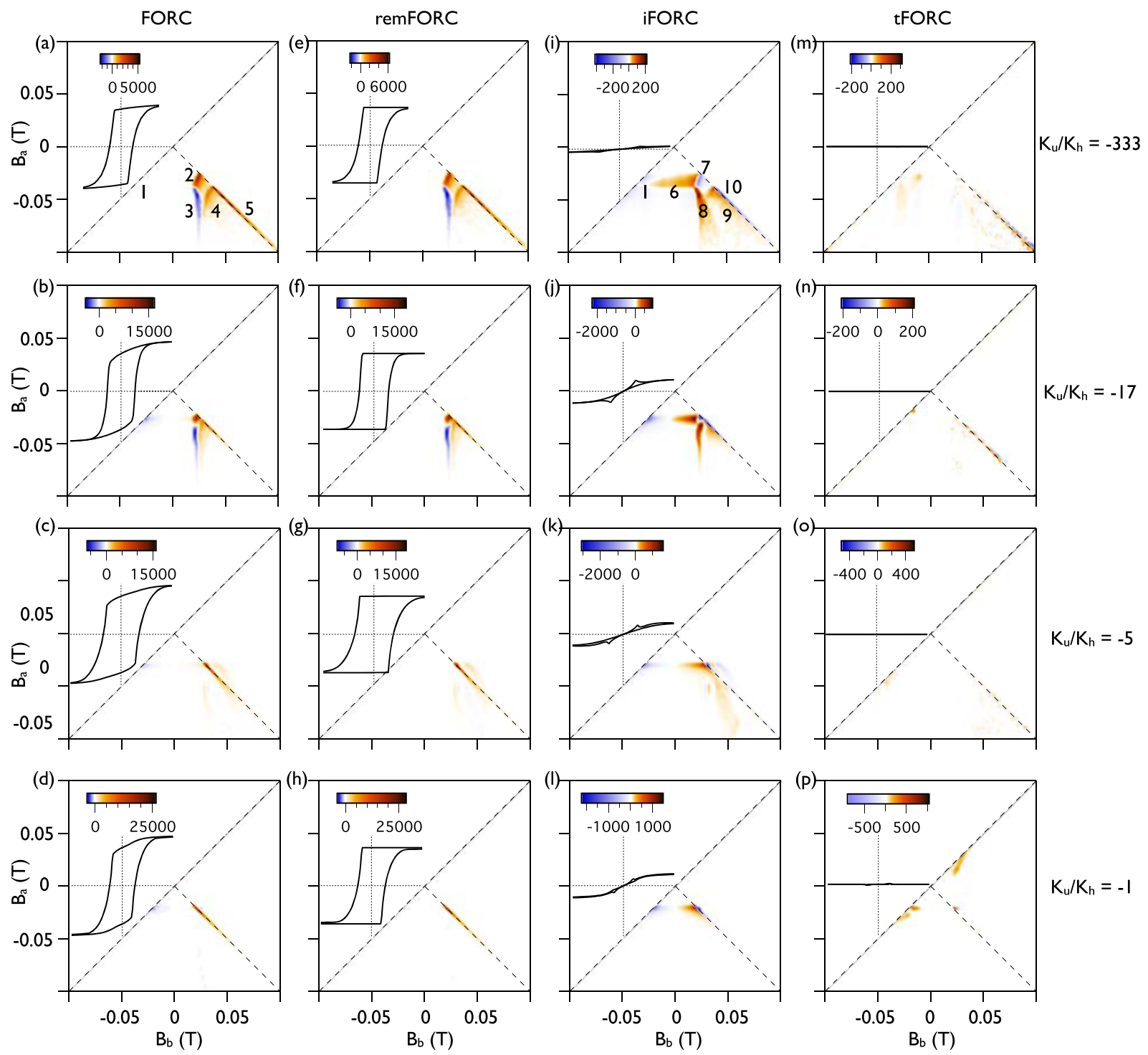

Figure 9. Processed (a-d) FORC, $(\mathrm{e}-\mathrm{h})$ remFORC, $(\mathrm{i}-\mathrm{l})$ iFORC, and $(\mathrm{m}-\mathrm{p})$ tFORC diagrams for randomly oriented non-interacting hexagonal particles with out-ofplane/in-plane anisotropy ratios (a, e, i m) $-333,(b, f, j, n)-17,(c, g, k, o)-5$, and $(\mathrm{d}, \mathrm{h}, \mathrm{l}, \mathrm{p})-1$. Insets are simulated hysteresis loops. Axis range for all insets is $M= \pm 1$ (vertical) and $B= \pm 0.1 \mathrm{~T}$ (horizontal). Magnetization values are normalized to $M_{\mathrm{s}}=1$.

remFORC diagram is less "teardrop" shaped compared to the uniaxial case (Figure 6f). For higher packing fractions, differences are less pronounced between uniaxial and cubic cases (Figures 6g, 6h, 7g, 7h). A weak positive signal along the $-B_{\mathrm{u}}$ axis (outside the remanence region) appears for strongly interacting particles (labelled $\mathrm{P}^{*}$ in Figure $7 \mathrm{~h}$ ).

\subsection{3. iFORCs}

For noninteracting particles, $M_{\mathrm{i}}\left(B_{\mathrm{a}}<0, B_{\mathrm{b}}\right)$ has a more pronounced maximum in positive measurement fields (Figure $3 \mathrm{c}$ ) than the noninteracting uniaxial case (Figure 2c), followed by a discontinuous change in slope at $B_{\mathrm{b}}=-B_{\mathrm{a}}$. Lower-branch-subtracted iFORCs have two large negative peaks and a smaller positive peak (Figure $3 \mathrm{c}$ inset). For strongly interacting particles, the discontinuous change in slope at $B_{\mathrm{b}}=-B_{\mathrm{a}}$ disappears, and a clear difference in slope at the beginning of each iFORC appears for positive reversal fields (Figure 3g). Lower-branch-subtracted iFORCs have two positive peaks (Figure 3g inset). Processed iFORC diagrams (Figures 7i-7l) have distinct positive and negative signals that evolve with increasing packing 
fraction. For noninteracting particles (Figure 7i), a clear N-P-N signature is seen, with negative and positive background signals and a strong negative central ridge. This pattern is distinct from that for noninteracting uniaxial particles (Figure 6i): The positive background signal extends to increasingly negative $B_{\mathrm{u}}$ values with increasing $B_{\mathrm{c}}$, whereas for uniaxial particles, the positive background signal tends to $B_{\mathrm{u}}=0$ with increasing $B_{\mathrm{c}}$. For $5 \%$ packing (Figure $7 \mathrm{j}$ ), the N-P-N pattern becomes similar to that observed in uniaxial particles with a comparable packing fraction (Figure $6 \mathrm{j}$ ). For $40 \%$ packing (Figure $7 \mathrm{k}$ ), a change to an N-P-N-P pattern is observed, which is broadly similar to that in uniaxial particles (Figure 6k). The change from the N-P-N to $\mathrm{N}-\mathrm{P}-\mathrm{N}-\mathrm{P}$ pattern coincides with the change from negative to positive lower-branch-subtracted iFORCs (Figures 3c, 3g insets), with the crossover occurring between $10 \%$ and $20 \%$ packing. For $74 \%$ packing (Figure 7l), iFORC features become weak and poorly resolved within simulation noise. The strongest feature is the positive signal $\left(\mathrm{P}^{*}\right)$ along the positive $B_{\mathrm{u}}$ axis.

\subsection{4. tFORCs}

Both raw (Figures $3 \mathrm{~d}$ and $3 \mathrm{~h}$ ) and processed (Figures $7 \mathrm{~m}-7 \mathrm{p}$ ) tFORCs for cubic particles have similar features as uniaxial particles at comparable packing fractions, except that the bilobate peaks for $74 \%$ packing are more intense and better defined for the cubic case (Figure $7 \mathrm{p}$ ).

\subsection{Results for Chains of Uniaxial Particles}

\subsubsection{FORCs}

Processed FORC diagrams for a range of chain collapse factors are summarized in Figure 8. Conventional FORC diagrams (Figures 8a-8c) reproduce the results of Harrison and Lascu (2014), with the wellestablished evolution from central ridge (Egli et al., 2010; Newell, 2005) to winged (Chen et al., 2007; Li et al., 2012) structures with increasing chain collapse. A prominent negative feature along the $-B_{\mathrm{u}}$ axis is visible in all cases.

\subsection{2. remFORCs}

Both straight and collapsed chains have $M_{\mathrm{rem}}\left(B_{\mathrm{a}}>0, B_{\mathrm{b}}\right)=$ constant $=M_{\mathrm{rs}}$ (Figure $4 \mathrm{~b}$, Figure $8 \mathrm{f}$ inset). The processed remFORC diagram for straight chains has an N-P-P structure, with a negative background signal, a positive background signal, and a positive central ridge (Figure $8 \mathrm{~d}$ ). This is superficially similar to the pattern observed for noninteracting cubic particles (Figure 7e). The negative background signal is absent for collapsed chains (e.g., $c=0.6$; Figure 8e). Instead, a broad, vertically spread low coercivity positive signal and a positive central ridge are observed (Figure $8 f$ ).

\subsection{3. iFORCs}

For straight chains, $M_{\mathrm{i}}\left(B_{\mathrm{a}}<0, B_{\mathrm{b}}\right)$ has a more pronounced maximum in positive measurement fields (Figure 4c), more similar to the noninteracting cubic case (Figure 3c) than the noninteracting uniaxial case (Figure 2c). Lower-branch-subtracted iFORCs have two large negative peaks (Figure 4c inset) but lack the intermediate positive peak for the cubic noninteracting case (Figure 3c). Processed iFORC diagrams (Figure 8g) have an N-P-N structure, similar to that of the noninteracting uniaxial case (Figure 6i). With increasing chain collapse, the positive background feature below the $B_{\mathrm{c}}$ axis becomes more distorted, and an additional positive feature appears above the $B_{\mathrm{c}}$ axis (Figure $8 \mathrm{~h}$ ), which is clearly different from both the randomly packed uniaxial and cubic cases (Figures $6 \mathrm{k}$ and $7 \mathrm{k}$ ).

\subsection{4. tFORCs}

Straight chains have zero tFORC signal (Figures $4 \mathrm{~d}$ and $8 \mathrm{j}$ ). Like the processed tFORC diagrams for interacting particles (Figures $6 \mathrm{~m}-6 \mathrm{p}$ ), collapsed chains have a bilobate distribution in the two transient regions that bound the remanence region (Figures $8 \mathrm{k}$ and 81 ). Rather than the two lobes emerging gradually from the origin, the lobes form "in-place" and slightly increase in strength with increasing chain collapse.

\subsection{Results for Non-Interacting Hexagonal Particles}

\subsubsection{FORCs}

Processed diagrams for noninteracting hexagonal particles with a range of $K_{\mathrm{U}} / K_{\mathrm{H}}$ ratios are summarized in Figure 9. For high values of $\left|K_{\mathrm{U}} / K_{H}\right|$ (moments strongly restricted to lie within the basal plane), complex FORC diagrams are predicted, with a mixture of positive and negative background and ridge features (labeled 1-5 in Figure 9a). Feature 1 is a weak negative signal close to the $-B_{\mathrm{u}}$ axis (barely visible in Figure 9a, but clearer in Figure 9b). Feature 2 is an elongated positive background signal, which extends below the $B_{\mathrm{c}}$ axis and merges with a highly elongated negative signal that extends in the $-B_{\mathrm{a}}$ direction (Feature 3). Feature 4 is a second elongated positive background signal to the right of Feature 3 . Feature 5 
is a positive central ridge signal. Features 2 and 5 become less elongated, and less separated, as $\left|K_{\mathrm{U}} / K_{\mathrm{H}}\right|$ decreases (Figure 9b). A dramatic change in FORC pattern occurs for $\left|K_{\mathrm{U}} / K_{\mathrm{H}}\right| \leq 5$ (Figure 9c): Features 2-4 disappear entirely, leaving behind only a paired negative Feature 1 and a positive central ridge (Figure 9d).

3.4.2. remFORCs

All remFORC simulations for particles with hexagonal anisotropy have $M_{\mathrm{rem}}\left(B_{\mathrm{a}}>0, B_{\mathrm{b}}\right)=$ constant $=M_{\mathrm{rs}}$ (Figure 5b, Figures 9e-9h insets). Processed remFORC diagrams differ only from the corresponding conventional FORC diagrams in the absence of negative Feature 1 (Figures 9e-9h).

\subsection{3. iFORCs}

Raw iFORCs have a distinctive double-peak structure in positive measurement fields (Figure 5c). Processed iFORC diagrams have a complex structure with positive and negative background and ridge features (labeled 6-10 in Figures 9i-9l). The negative Feature 1 that was absent in the remFORC diagram appears in the iFORC diagram. Feature 6 is a positive background signal that is present in the FORC signal, although its weak intensity makes it barely visible on the color scale used for Figure 9a. Feature 6 is absent from the remFORC signal (Figure 9e). A sharp change in iFORC pattern occurs for $\left|K_{\mathrm{U}} / K_{\mathrm{H}}\right| \leq 5$ (Figure 9k): Features 7-9 disappear entirely, leaving an N-P-N structure similar to that in noninteracting uniaxial particles (Figure 91).

3.4.4. tFORCs

All noninteracting particles with hexagonal anisotropy have zero tFORC signals for all $K_{\mathrm{U}} / K_{\mathrm{H}}$ ratio values.

\section{Discussion}

4.1. Physical Origins of remFORC, iFORC, and tFORC Signals

4.1.1. Random Packing of Uniaxial and Cubic Particles

Our simulations demonstrate how positive and negative background and ridge signals partition into either remFORC, iFORC, or tFORC signals according to their physical origin. The positive central ridge in a FORC diagram for noninteracting uniaxial SD particles (Figure 6a) appears exclusively in the remFORC signal (Figure 6e), consistent with its origin resulting from irreversible switching (Newell, 2005). Negative and positive background FORC signals, on the other hand, appear exclusively in the iFORC signal (Figure 6i), consistent with their origin in different reversible slopes of upper and lower hysteresis branches (Newell, 2005). A negative central ridge in the iFORC signal is associated with the discontinuous change in slope of $M_{\mathrm{i}}$ at $B_{\mathrm{b}}=-B_{\mathrm{a}}$ (Figure 2c). The resulting N-P-N iFORC structure was treated as diagnostic of weakly interacting SD behavior by Zhao et al. (2017).

FORC, remFORC, and iFORC signals of noninteracting cubic particles are distinct from those of uniaxial particles (Figures 7a, 7e, 7i). A conventional FORC diagram for cubic particles has an N-N-P-P structure with two negative background features, a positive background feature, and a positive central ridge (Figure 7a). The first negative feature appears exclusively in the iFORC diagram (Figure 7i), which demonstrates that, like the uniaxial case, it is caused by the difference in reversible slope of hysteresis branches for cubic particles. The second negative feature, however, appears exclusively in the remFORC diagram (Figure 7e), which demonstrates that it is associated with irreversible switching events. Unlike the uniaxial case, where there is just one easy axis and two corresponding hysteresis branches, cubic particles have four $<111>$ easy axes and eight corresponding hysteresis branches. For certain combinations of applied field and particle orientation, asymmetric switching between different easy axes yields both positive and negative background signals (Valdez-Grijalva \& Muxworthy, 2019). A portion of the positive background signal can, therefore, be attributed to asymmetric switching between different easy axes (Figure 7e). The remaining portion appears in the iFORC diagram (Figure 7i), and, like the uniaxial case, results from the difference in reversible slopes of different hysteresis branches. The positive ridge signal in the remFORC diagram is associated with symmetric switching involving a single easy axis (Figure 7e). This signal is mirrored by a negative ridge in the iFORC diagram (Figure 7i), which leads to a similar (yet distinct) N-P-N structure to that of the uniaxial case.

With increasing interactions, the distinction between cubic and uniaxial particles becomes less pronounced. Weak negative background features appear in the remFORC diagram for both uniaxial (Figure 6f) and cubic (Figure 7f) particles, broadening of the remFORC signal merges the background and ridge signals, and the 


\section{Uniaxial fcc \\ $74 \%$ packing, high anisotropy}

(a) + I T

(b) $+300 \mathrm{mT}$

(c) $+100 \mathrm{mT}$
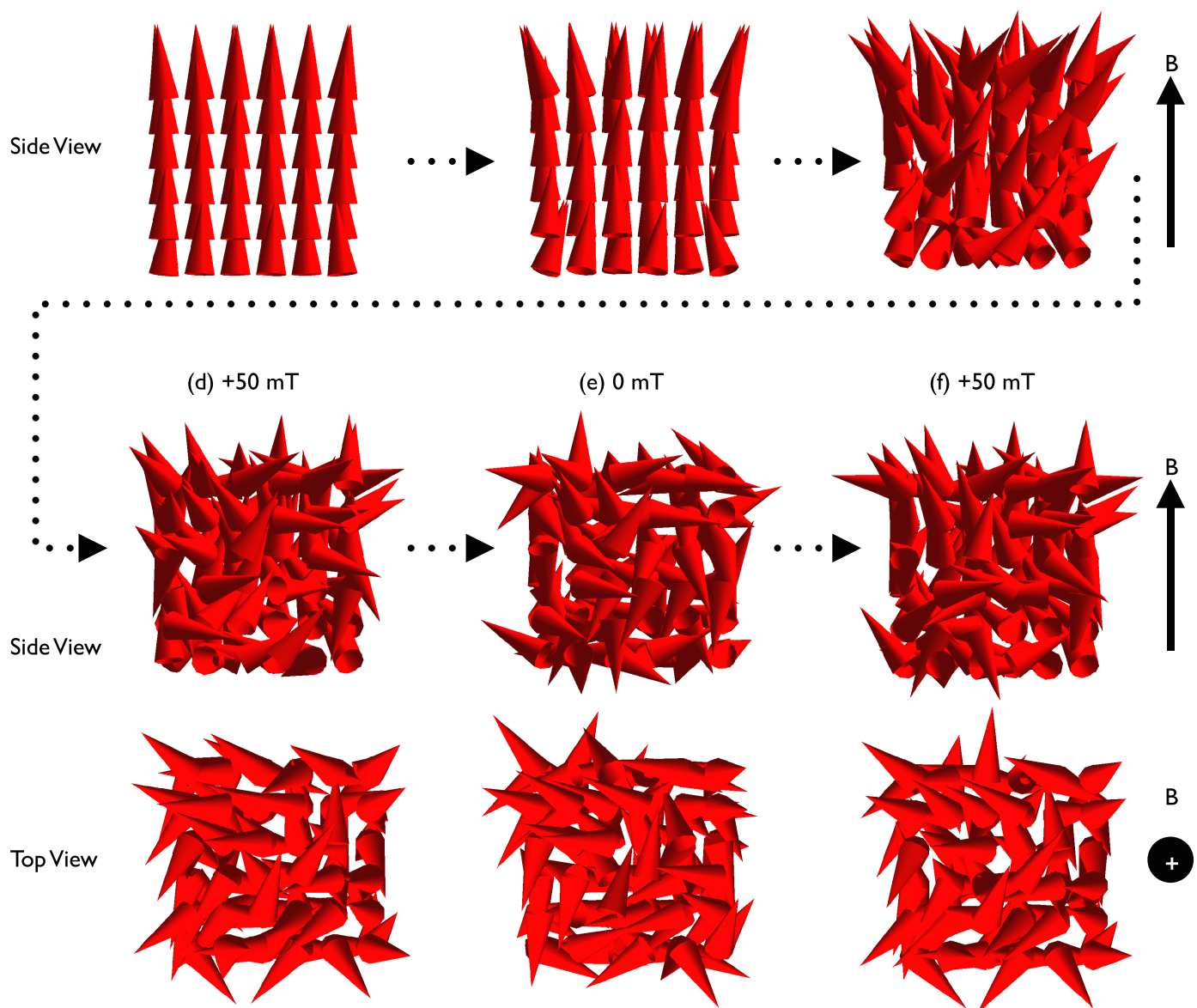

(e) $0 \mathrm{mT}$

(f) $+50 \mathrm{mT}$
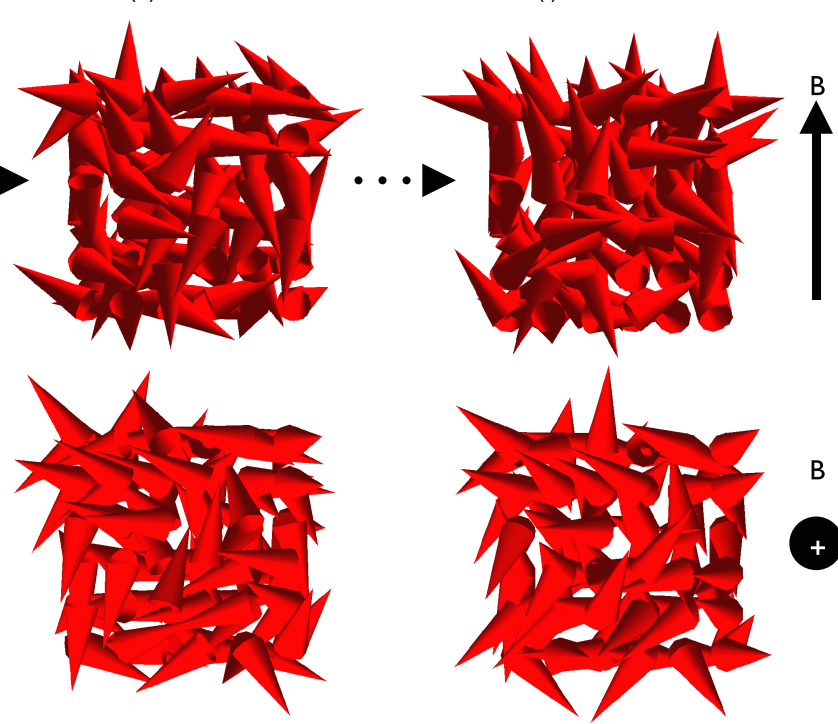

B

Figure 10. Simulation snapshots for 108 uniaxial particles arranged in a $3 \times 3 \times 3$ cubic closed packed array with $74 \%$ packing fraction. Simulations were performed as a function of decreasing applied field, from (a) $1 \mathrm{~T}$ to (b) $300 \mathrm{mT}$, to (c) $100 \mathrm{mT}$, to (d) $50 \mathrm{mT}$, and (e) $0 \mathrm{mT}$. The field was then increased from $0 \mathrm{mT}$ back to $50 \mathrm{mT}$ (f). The field direction is indicated by the arrow. The snapshot images represent the development of flower states in high fields $(\mathrm{a}-\mathrm{c})$. Below $100 \mathrm{mT}$, a large proportion of particles switch to their reversed state as a result of strong magnetostatic interactions with their neighbors (d-e). This leads to development of domain superstructures reminiscent of the multivortex states observed in large particles (Lascu et al., 2018). The difference in magnetic state observed at $50 \mathrm{mT}$ in (d) and (f) corresponds to the transient magnetization measured in a tFORC.

distinction between N-P-N iFORC patterns for uniaxial (Figure 6j) and cubic (Figure 7j) cases becomes less obvious. Both uniaxial and cubic particles develop bilobate tFORC signals with increasing interactions. In the absence of thermal activation, transient hysteresis is primarily associated with irreversible changes in magnetization driven by self-demagnetizing fields (Fabian, 2003). Zhao et al. (2017) associated bilobate structures in tFORC diagrams with vortex state nucleation and annihilation, in agreement with both theoretical modeling and empirical observations of materials dominated by vortex states (Pike \& Fernandez, 1999; Dumas et al., 2007; Roberts et al., 2017; Valdez-Grijalva et al., 2018; Lascu et al., 2018). Our simulations demonstrate that analogous effects are seen in strongly interacting clusters of SD particles, due to formation of flux closure structures driven by the interparticle dipole-dipole interactions. Snapshots of magnetic configurations obtained in a cubic-close-packed cluster of uniaxial particles are shown in Figure 10. Starting in a field of $+1 \mathrm{~T}$ (Figure 10a), the cluster is in a saturated state with each particle moment aligned closely to the field. As the field is reduced to $+300 \mathrm{mT}$ (Figure 10b), the cluster adopts a "super-flower" state, analogous to the micromagnetic flower state observed in single particles just below the threshold size for vortex nucleation (Schabes \& Bertram, 1988; Williams \& Dunlop, 1989, 
1995). With further field reduction to $+100 \mathrm{mT}$ (Figure 10c) and $50 \mathrm{mT}$ (Figure 10d), flowering becomes more pronounced and interaction-driven switching of particles into reversed states begins. At remanence (Figure 10e top view), creation of a flux-closure structure loosely resembles a super-vortex (Harrison et al., 2002). Imperfect moment alignment is due to competition between anisotropy and interaction fields. Simulations with reduced anisotropy demonstrate a more obvious super-vortex due to dominant interaction fields. If the field is ramped from zero to $+50 \mathrm{mT}$, direct comparison can be made between magnetic states on the upper hysteresis branch (Figure 10d) and on the zFORC (Figure 10f). The magnetization difference of these two snapshots is equal to the transient magnetization (cf. measurement points 2 and 4 in Figure 1).

Simulated FORC, remFORC, iFORC, and tFORC signals for weakly-to-moderately interacting SD particles agree well with key features observed experimentally by Zhao et al. (2017). For example, a floppy magnetic recording disk with interacting SD particles (Figure 4 of Zhao et al., 2017) has all of the characteristics predicted here for cubic particles with $5 \%$ packing fraction (Figures $7 \mathrm{~b}, 7 \mathrm{f}, 7 \mathrm{j}$, and $7 \mathrm{n}$ ). Experimental FORC results for densely packed, synthetic magnetite particles (Sigma Aldrich 637106-25G) are shown in Figure 11. Although these particles span the SD-SV size range (Figure 11b), many key features predicted for strongly interacting uniaxial and cubic SD particles are observed in the experimental data, including spreading of the FORC signal at low coercivity (Figure 11c), the shape and spreading of the remFORC signal (Figure 11d), the complex shape of the N-P-N-P iFORC structure (Figure 11e), and a bilobate tFORC distribution (Figure 11f). A positive feature close to the $-\mathrm{B}_{\mathrm{u}}$ axis in the experimental remFORC diagram is associated with viscous relaxation effects (Hu et al., 2018; Zhao et al., 2017), which are not modeled here. A prominent negative feature in the experimental tFORC diagram is less well reproduced by the simulations, although a weak negative feature is visible in a tFORC diagram for cubic particles (Figure 7o). Other differences between simulated and observed behavior are likely due a combination of factors, including (a) failure to account for thermal relaxation in the simulations, (b) differences between assumed and actual coercivity distributions, and (c) predominance of SV particles in the sample. The behavior of these densely packed particles has striking similarities to basalt samples with "PSD" magnetite (Figure 6 of Zhao et al., 2017), which have been attributed to vortex nucleation and annihilation. Although the presence of SV particles in our samples helps to explain this similarity, the simulations demonstrate that vortex states sensu stricto are not required in order to produce these patterns. The key physical driver is the presence of strong demagnetizing effects (either self-demagnetization for vortex nucleation/annihilation or dipole-dipole interactions for packed SD clusters).

A key observation for strongly interacting clusters is the pronounced $M_{\text {rem }}\left(B_{\mathrm{a}}>0, B_{\mathrm{b}}\right)$ decrease with increasing applied field (Figures $2 \mathrm{f}$ and $3 \mathrm{f}$ ). Having reduced the applied field gradually from a saturating value to zero (e.g., Figures 10a-10e), increasing the field to a positive value (e.g., Figure 10f) and then back to zero (not shown in Figure 10) results in a remanence drop. Our interpretation of this phenomenon is that intergrain magnetostatic interactions dominate, creating complex field-dependent energy surfaces, where field direction changes are no longer necessarily reversible; In the noninteracting SD case, energy surfaces are smooth and controlled only by magnetocrystalline anisotropy, which makes them relatively reversible.

4.1.2. Chains

For straight chains of particles, strong magnetostatic interactions along the chain axis produce collective switching behavior. The switching field of chains lies at the upper end of the coercivity distribution of individual particles that make up the chain. FORC and iFORC diagrams for straight chains (Figures 8a and 8g) resemble those for noninteracting uniaxial particles (Figures 6a and 6i). The remFORC diagram, however, has an N-P-P structure similar to that observed for noninteracting cubic particles (Figure 7e). Unlike the cubic case, where the negative background remFORC signal is related to switching between different easy axes (section 4.1.1), for straight chains this signal is related to partial switching of chains at intermediate fields (Figure 12). The lowest energy state of a straight chain is fully magnetized either parallel or antiparallel to the chain axis (Figure 12a). Moment rotation in negative reversal fields initiates at the ends of the chain (Figure 12b), where interaction fields that keep moments aligned with the chain axis are reduced (particles on the ends of the chain have a single nearest neighbor, whereas those in the center have two). If all particles have similar coercivity, then switching of the ends of the chains would occur simultaneously through a fanning mechanism (Jacobs \& Bean, 1955). If particles in the center of the chain have sufficiently high 
(a) SEM

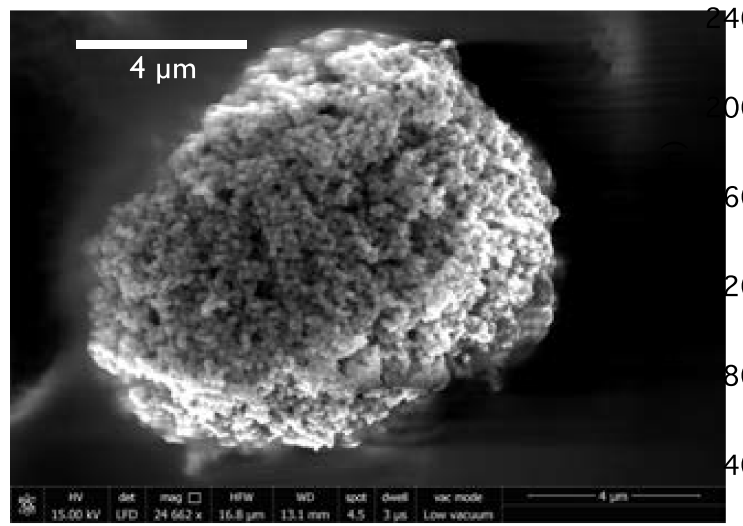

(c) FORC

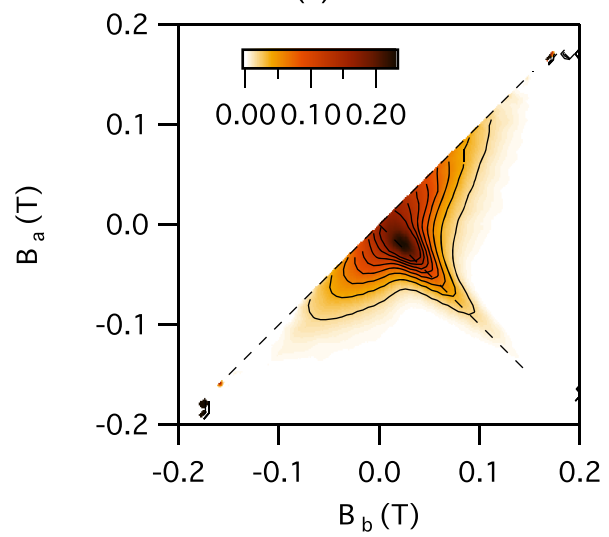

(e) iFORC

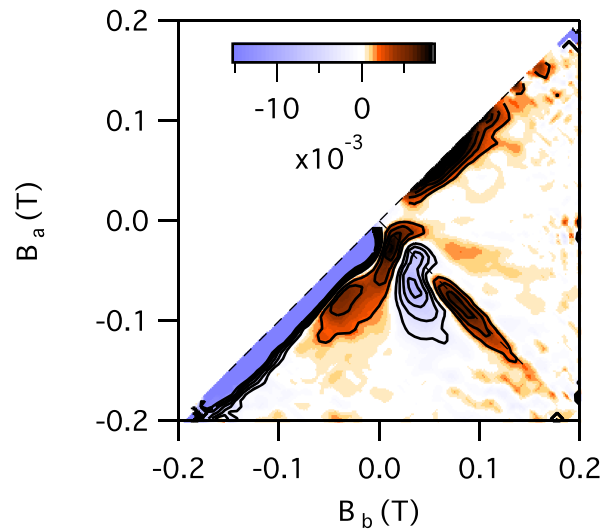

(b) Size Distribution

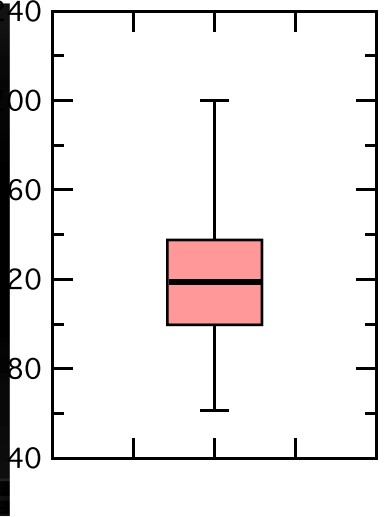

(d) remFORC

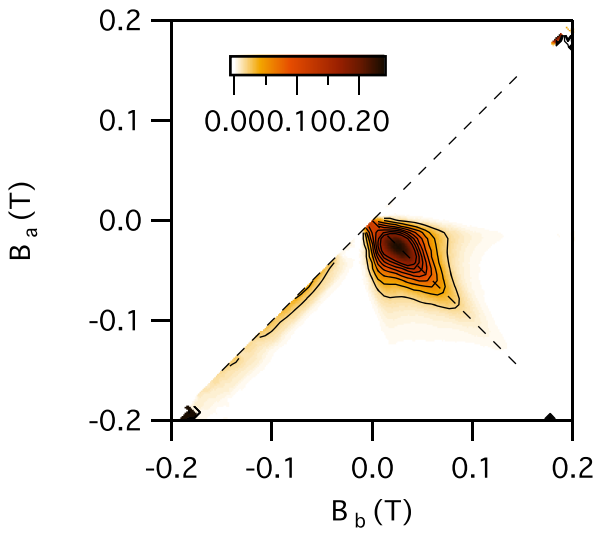

(f) tFORC

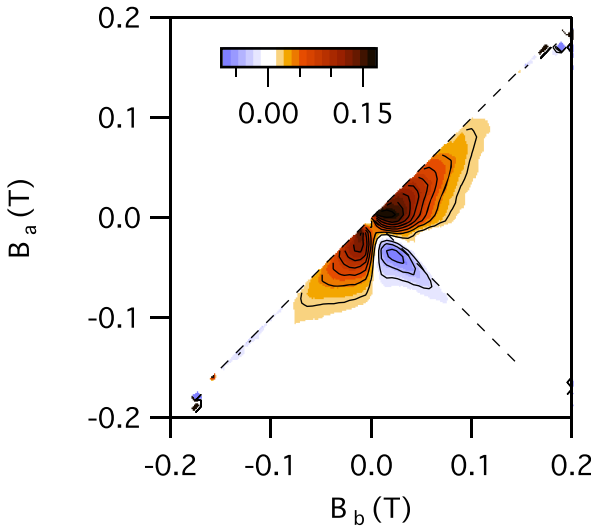

Figure 11. (a) Secondary electron scanning electron microscope image of the analyzed synthetic magnetite sample (Sigma Aldrich 637106-25G). (b) Box-whisker plot of particle diameter distribution measured manually from a random sampling of 60 particles. Thick horizontal line indicates the median $(120 \mathrm{~nm})$. Whiskers indicate the 2nd and 98th percentiles, which vary from 50 to $200 \mathrm{~nm}$. The box represents the 25th to 75 th percentiles. The sizes span the SD-SV range, with most in the SV size range. (c) FORC, (d) remFORC, (e) iFORC, and (f) tFORC diagrams for the synthetic magnetite sample measured using the protocol of Zhao et al. (2017).

coercivity, however, only moments at the ends of the chain switch (Figure 12c). A partially switched chain (Figure 12d) is metastable and needs a much smaller positive measurement field to switch it back to its saturated state (Figure 12e). Larger reversal fields eventually lead to full switching of the chain (Figure 12f), potentially via a series of intermediate states. The fully reversed remanence state is shown in 


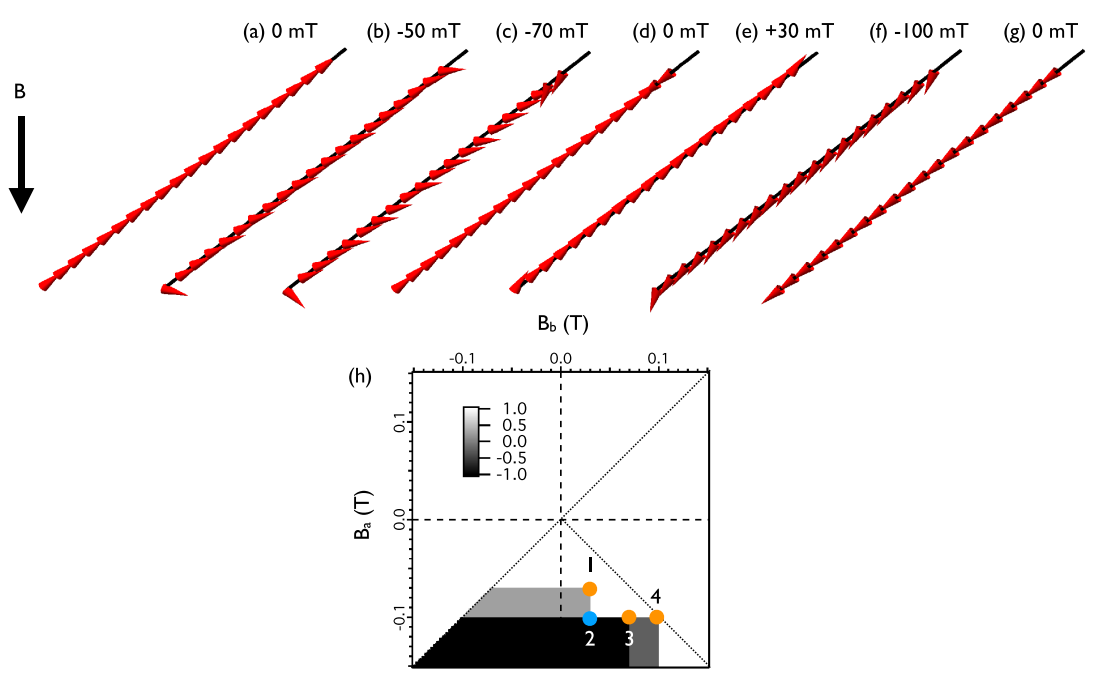

Figure 12. Partial switching of straight chains provides an explanation for negative and positive background remFORC signals observed in Figure 10d. (a) The initial remanence state is magnetized uniformly along the chain length. (b) $B_{\mathrm{a}}=-50 \mathrm{mT}$. Rotation initiates at the ends of the chain, where the local interaction field is reduced. (c) Switching occurs at the upper end of the chain in reversal field $B_{\mathrm{a}}=-70 \mathrm{mT}$. Switching does not propagate along the chain due to the presence of higher coercivity particles in the central portion. (d) remFORC state acquired after application of a $-70 \mathrm{mT}$ field. (e) Switching back of the end of the chain occurs in $B_{\mathrm{b}}=+30 \mathrm{mT}$. (f) Full switching of the chain occurs after $B_{\mathrm{a}}=-100 \mathrm{mT}$. (g) Final remFORC state. (h) Schematic illustration of $M_{\mathrm{rem}}$ as a function of $B_{\mathrm{a}}$ and $B_{\mathrm{b}}$. Points where positive and negative contributions to the remFORC distribution are made are shown as orange and blue dots, respectively.

Figure 12g. A schematic illustration of $M_{\text {rem }}$ as a function of $B_{\mathrm{a}}$ and $B_{\mathrm{b}}$ is shown in Figure $12 \mathrm{~h}$ for a chain in a single partially switched intermediate state. Locations of nonzero remFORC contributions (equation (5)) are highlighted, which demonstrates how the combined partial and full switching events lead to two positive background signals (1 and 3), a negative background signal (2), and a positive ridge signal (4).

The tFORC signal for collapsed chains has the same bilobate pattern observed in randomly packed clusters and is related to formation of similar flux-closure structures driven by dipole-dipole interactions (section 4.1.1; Figure 10). However, collapsed chains retain a stronger noninteracting uniaxial component in FORC, remFORC, and iFORC signals than randomly packed clusters with comparable tFORC signals (cf. Figures $6 \mathrm{c}, 6 \mathrm{~g}, 6 \mathrm{k}, 60$ with Figure $8 \mathrm{~b}, 8 \mathrm{e}, 8 \mathrm{~h}, 8 \mathrm{k}$ ). This distinguishing feature is most obvious in the iFORC diagram, which retains a clear N-P-N structure that is more similar to Figure $6 \mathrm{j}$ than Figure $6 \mathrm{k}$. In addition, the iFORC diagram for collapsed chains has an additional positive signal above the $B_{\mathrm{c}}$ axis that is not present in Figure 6j or Figure 7j. Comparison of a predicted iFORC diagram for collapsed chains with an experimental iFORC diagram for a magnetofossil-rich sediment from the onset of the PETM (Chang et al., 2018) is shown in Figure 13. Both the strong N-P-N structure and the additional positive feature are present. The positive signal along the $+B_{\mathrm{u}}$ axis in the experimental iFORC diagram is not reproduced in simulations. Although similar signals are predicted for strongly interacting clusters (e.g., Figures 61, 7l; labeled P*), these are thought to be simulation artefacts (see section 4.2) that coincidentally mimic real physical processes.

\subsubsection{Hexagonal Anisotropy}

FORC, remFORC, and iFORC signals for particles with hexagonal anisotropy are complex and change fundamentally as a function of $\left|K_{\mathrm{U}} / K_{H}\right|$. The physical origin of complex FORC behavior for hexagonal particles was discussed preliminarily by Harrison et al. (2017) and will be expanded upon in a separate paper. The five signals observed in a FORC diagram for hexagonal particles with high $\left|K_{\mathrm{U}} / K_{H}\right|$ (Figure 9a) are virtually identical to those predicted by Valdez-Grijalva and Muxworthy (2019) for randomly oriented particles with cubic anisotropy. These signals were attributed by Valdez-Grijalva and Muxworthy (2019) to the availability of multiple easy axes in the cubic system. The physical origin of these signals in hexagonal particles is similarly related to the availability of multiple easy axes within the basal plane (Harrison et al., 2017). Negative signal 1 is partitioned into the iFORC component, and, like the uniaxial and cubic cases, it is caused by different reversible slopes of hysteresis branches. Like the cubic case, positive and negative background remFORC 


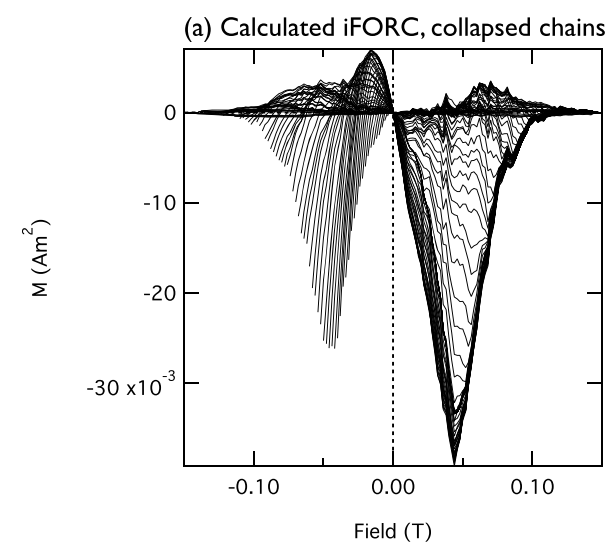

(b) Processed iFORC, collapsed chains
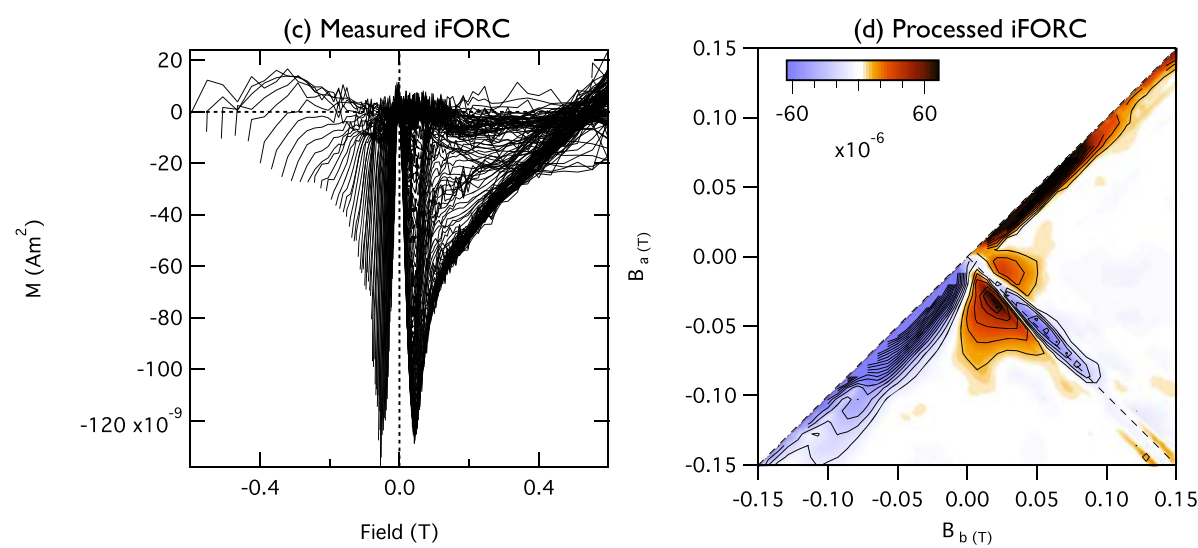

Figure 13. Simulated (a) raw curves and (b) processed iFORC diagram for fully collapsed chains of uniaxial particles. (c) Measured and (d) processed iFORC diagrams for a magnetofossil-rich sample (ODP Hole 1263C, section 14H-2A, interval 146-147 cm, at $335.67 \mathrm{~m}$ composite depth; Chang et al., 2018).

signals $(2,3,4)$ are caused by asymmetric switching between different easy axes. Positive ridge signal $(5)$ is related to symmetric switching involving the same easy axis. The $B_{\mathrm{c}}$ extent of the ridge is highly sensitive to I $K_{\mathrm{U}} / K_{H} \mid$, which becomes less prominent with lower $\left|K_{\mathrm{U}} / K_{H}\right|$, along with a smaller gap between positive signals 2 and 4 (Figures 9b, 9f, 9j, 9n).

The presence of multiple easy axes is evident in the raw iFORC signal (Figure 5c), which has a distinctive double-peak structure in positive measurement fields due to an intermediate easy axis that occurs for certain applied field and particle orientation combinations. Although this feature is not visible in the cubic case (Figure 3c), both hexagonal and cubic iFORC diagrams have a positive peak in the lower-branch subtracted iFORCs (Figures $3 \mathrm{c}$ and $5 \mathrm{c}$ insets), which suggests that this feature may also be diagnostic of multiple easy axes. Experimental confirmation of the anomalous double-peaked raw iFORC behavior is shown in Figure 14 for a MD hematite single crystal with field applied at $30^{\circ}$ to the basal plane (Iwaki, 1965).

The remarkably similar behavior of cubic and hexagonal particles demonstrates that it is the availability of multiple easy axes that produces the asymmetric positive and negative background features that are displaced negatively below the $B_{c}$ axis. Any nonuniaxial mineral is expected to have such features, but they are likely to be most pronounced in minerals dominated by magnetocrystalline anisotropy. Hematite is most often associated with such asymmetric FORC signals, which is unsurprising given that shape anisotropy is weak in hematite due to its low saturation magnetization. Figure $9 \mathrm{~b}$ with $\left|K_{\mathrm{U}} / K_{H}\right|=17$ is closest to the "kidney bean" shape often associated with hematite FORC diagrams (Brownlee et al., 2011; Carvallo et al., 2006; Carvallo \& Muxworthy, 2006; Church et al., 2016; Jovane et al., 2011; Liu et al., 2010; Martín-Hernández \& Guerrero-Suárez, 2012; Muxworthy et al., 2005), especially considering that we simulated populations of identical particles, rather than for coercivity distributions, which would further smear the signal. Examples of dominantly uniaxial central ridge behavior have also been documented for hematite (e.g., 
(a) Calculated iFORC, hexagonal

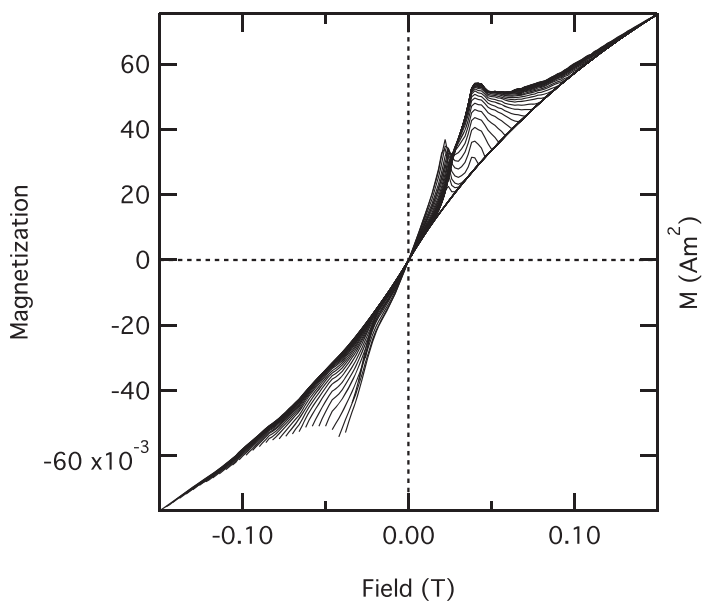

(b) Measured iFORC hematite single crystal

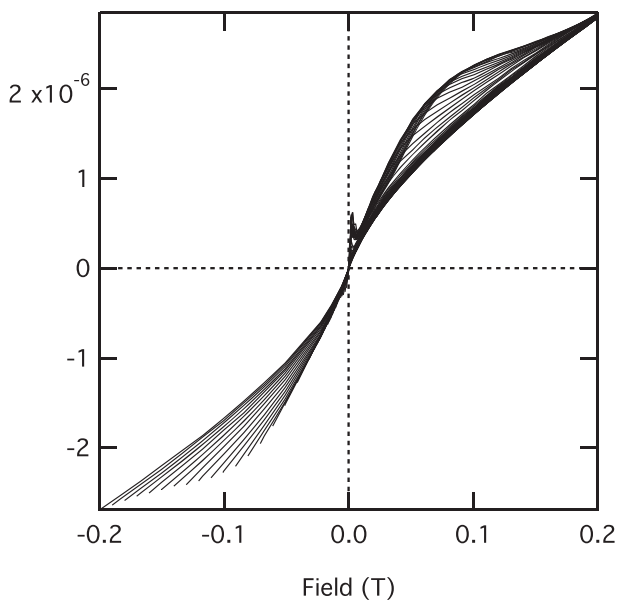

Figure 14. (a) Simulated iFORCs for randomly arranged particles with hexagonal anisotropy with a double peak for positive measurement fields. (b) Measured iFORCs for a single crystal of hematite. The sample is a 5-mm fragment of natural specularite crystal from Mt Shimotoku, Okayama Prefecture, Japan, from the collection of the Geological Museum of the Geological Survey of Japan (Registration number A31-36426). The magnetic properties of hematite crystals from this locality have been reported by Iwaki (1965).

Jiang et al., 2016; Pariona et al., 2016; Roberts et al., 2006). A transition to uniaxial switching behavior is predicted here for samples with low $\left|K_{\mathrm{U}} / K_{H}\right|$ (Figures 9d, 9h, 9l, 9p). Below a critical $\left|K_{\mathrm{U}} / K_{H}\right|$ value, symmetric switching between a single easy axis is achieved by rotating spins out of the basal plane, rather than by rotating spins within the basal plane via an intermediate easy axis. Low $\left|K_{U} / K_{H}\right|$ can be achieved by either lowering $K_{\mathrm{U}}$ or by increasing $K_{\mathrm{H}}$. Lowering $K_{\mathrm{U}}$ is unlikely because its intrinsically high value is related to the fundamental anisotropy of the hematite crystal structure. Increasing $K_{\mathrm{H}}$ (e.g., through magnetoelastic coupling to basal plane stress) is more easily achievable. Hence, the observation of asymmetric multiaxial vs symmetric uniaxial switching behavior in hematite may be related to a fundamental difference in the balance of in-plane versus out-of-plane anisotropy and may yield insight into the stress state of hematite particles.

Despite its cubic symmetry, SD magnetite is typically dominated by uniaxial shape anisotropy, and is therefore dominated by central ridge signals. Greigite, on the other hand, typically grows in sedimentary environments as equidimensional crystals with cuboctahedral symmetry (Roberts et al., 2011), and commonly gives rise to the asymmetric combination of positive and negative background features predicted here for hexagonal particles and by Valdez-Grijalva and Muxworthy (2019) for cubic particles. Similar arguments apply to pyrrhotite, which also has highly asymmetric FORCs (Horng, 2018; Kars \& Kodama, 2015a, 2015b; Larrasoaña et al., 2007; Roberts et al., 2010; Roberts et al., 2018; Weaver et al., 2002; Wehland et al., 2005).

\subsection{Simulation Artefacts?}

Some positive and negative features in the simulated results (labeled $\mathrm{P}^{*}$ and $\mathrm{N}^{*}$ in Figures 8 and 9 ) may be simulation artefacts. These features appear along the $+B_{\mathrm{u}}$ and $-B_{\mathrm{u}}$ axis, a region that is well-known for socalled "first-point artefacts." In an experimental context, the first-point artefact is caused by the first point of each FORC measurement being offset from the rest of the FORC due to instrumental measurement factors (the first point is measured in static mode, and subsequent points are made in field-sweep mode). In a simulation context, first-point artefacts may be created by the inevitably large applied field jump from the reversal field to zero to measure the first point in a remFORC. Ideally, the field would be stepped to zero gradually from the reversal field, as is the case for FORC simulations, but this would be prohibitively expensive for remFORC computations. A large difference is observed between the remanence value obtained after a single large step from the reversal field to zero compared with that obtained during subsequent measurements (Figure 15a). The discrepancy increases with increasing $\left|B_{\mathrm{a}}\right|$ and results in a steep initial downturn in remFORC curves that mimics the viscous relaxation that is often observed experimentally in the same region 
(a) Option I

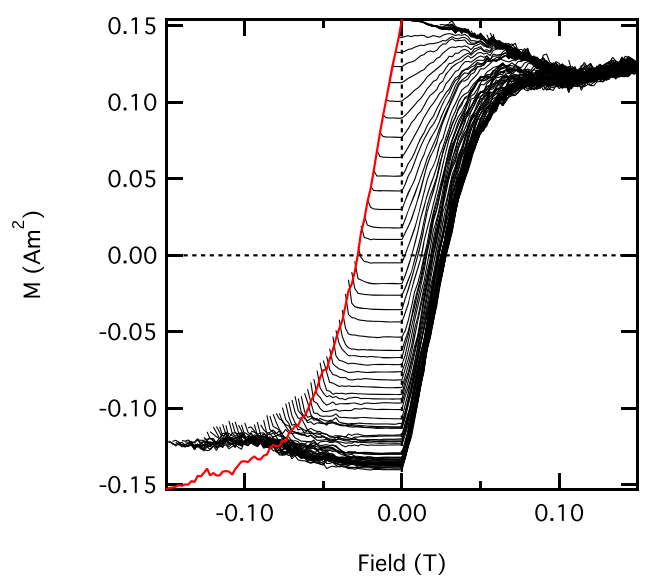

(c) First point included

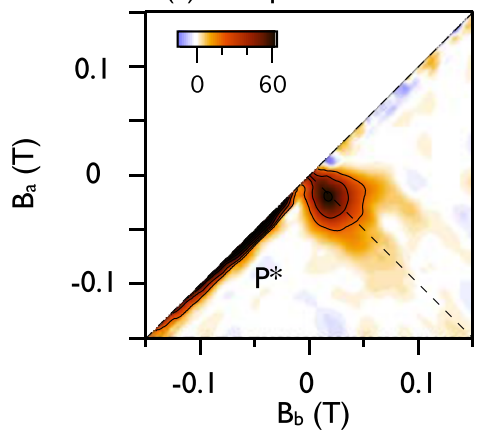

(d) First point removed

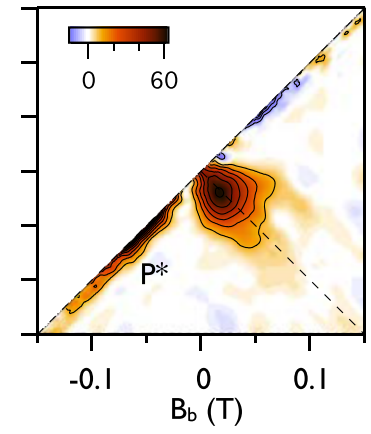

(b) Option 2

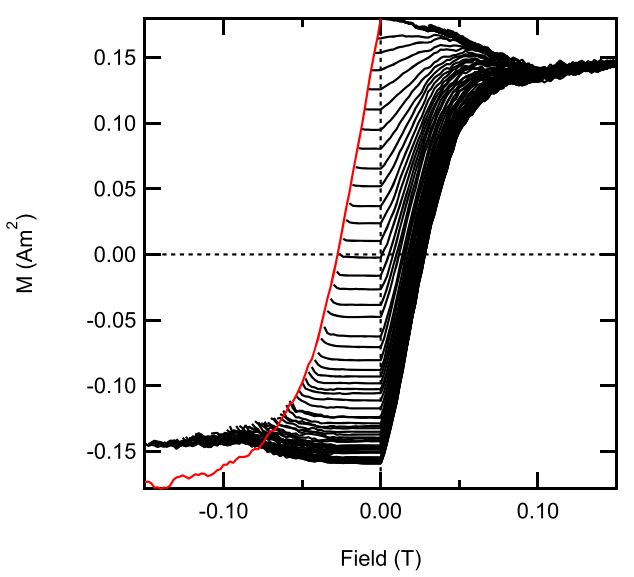

(e) First point included

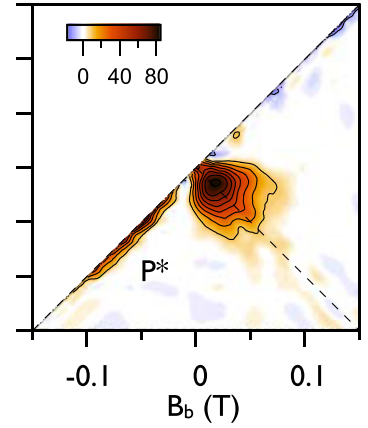

(f) First point removed

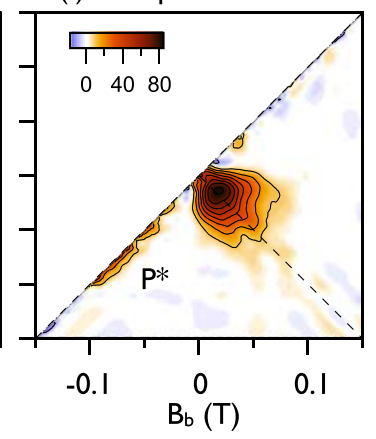

Figure 15. Simulated remFORCs for randomly packed cubic particles with 74\% packing fraction using (a) Option 1 and (b) Option 2 of the remFORC simulation protocol. In Option 1, the simulation is initialized at the reversal field using the starting configuration obtained at the corresponding point of the upper branch of the hysteresis loop. In Option 2, the simulation is initialized at remanence, using the starting configuration obtained from the FORC with corresponding reversal field. Back-field remanence values obtained from the FORCs are shown as red curves. Note the larger "first-point artefact" in (a). Processed remFORCS for Option 1 are shown both with (c) and without (d) the first point included. Similarly, for Option 2, in (e) and (f). The residual P* signal is evidence of a "field cycling" effect, analogous to the "thermal cycling" effect of Fabian and Shcherbakov (2004).

(e.g., Hu et al., 2018) and leads to real remFORC signals in this area (Figure 11d). The effect is most pronounced when Option 1 is used to determine the remFORC (Figure 15a). The effect is reduced for Option 2 (Figure 15b). This is because the starting remanence for Option 2 is obtained by gradually stepping the field from the reversal field to zero during the initial FORC simulation. Further reduction of $\mathrm{P}^{*}$ and $\mathrm{N}^{*}$ features in processed FORC diagrams can be obtained by removing the first point of each curve from the data set prior to processing (Figures 15c-15f).

Despite the discussion above, relaxation of remanence is observed well beyond the first point of each remFORC, and a $\mathrm{P}^{*}$ signal is observed in processed remFORC diagrams even when the first point is omitted prior to processing (Figure 15f). The relaxation observed during iterative back-field cycling resembles that observed during iterative thermal cycling of MD grains (Fabian \& Shcherbakov, 2004) who explained this phenomenon with a statistical theory involving a stochastic transition matrix, which describes the probability that the magnetic state of an MD particle transforms into a different one during a thermal cycle iteration. The mathematical formalism of Fabian and Shcherbakov (2004) is general (although transformation matrix details may differ for MD particles versus interacting SD clusters). The fact that there is good agreement between observed and simulated remFORC signals outside the remanence region (cf. Figures 11d and 15e) raises the possibility that the evolution of remanence during iterative back-field cycling is not an artefact but a real phenomenon that relates to statistical equilibration of the probability density of magnetic states within strongly interacting clusters. The field-cycling history for each $B_{\mathrm{b}}$ step contains information about all previous field-cycling steps for a particular reversal field $B_{\mathrm{a}}$, which is then carried forward to the 
next measurement step. The system is only "cleaned" prior to applications of the next reversal field. For strongly interacting SD systems contributions to the remFORC diagram in this region will depend on field step size, both in terms of the distribution of intensity and their position, for both models and experiments. This "field cycling" effect, i.e., minor hysteresis loops, is analogous to the "thermal cycling" effect described by Fabian and Shcherbakov (2004) for MD particles and provides an alternative to SP behavior as an explanation for the $\mathrm{P}^{*}$ signals observed commonly in remFORC diagrams.

\section{Conclusions}

Micromagnetic simulations for the extended FORC protocol of Zhao et al. (2017) demonstrate how the total FORC signal is partitioned between remFORC, iFORC, and tFORC signals. This work provides the first theoretical framework for predicting and interpreting these new FORC-type diagrams. Despite the additional time required to measure these FORC-type diagrams, our simulations demonstrate their additional interpretive power by linking each observed signal to a different physical aspect of the magnetization process. Good agreement between simulated and observed behavior is found for a range of samples. In particular, the spreading and shape of remFORC distributions, the transition from the N-P-N to N-P-N-P structure in the iFORC diagram, and generation of bilobate tFORC distributions are reproduced accurately in strongly interacting SD clusters. These signals also appear to be a good analog for "PSD" samples dominated by particles that lie in the SV/MV size range (Lascu et al., 2018; Roberts et al., 2017). Strong coupling between reversible and irreversible magnetization components is identified in strongly interacting clusters, which leads to a decreasing remanence trend with increasing magnetizing field. Appearance of strong negative signals in remFORC diagrams are linked to particles or chains with intermediate switching states. For individual particles, these intermediate states correspond to multiple easy axes and explain the characteristic "kidney"shaped FORC fingerprint of minerals such as hematite and pyrrhotite, which are dominated by multiaxial magnetocrystalline anisotropy. A transition to uniaxial switching in hexagonal particles is found below a critical value of the out-of-plane/in-plane anisotropy ratio. Similar fingerprints in minerals such as greigite are due to its common occurrence as equidimensional grains with limited shape anisotropy. For straight chains, intermediate states are achieved by partial switching when there is sufficient coercivity variation in particles along a chain. A distinct positive signal appears in the iFORC signature for collapsed chains of uniaxial particles, which may aid discrimination between biogenic and nonbiogenic signals in sediments. Good agreement between simulated and observed behavior means that this approach has merit for generating training data for machine-learning algorithms applied to automated detection and quantification of diagnostic features in FORC and FORC-like diagrams. With the increased complexity of information provided by the FORC-type diagrams of Zhao et al. (2017), we anticipate that development of machine-learning algorithms for automated FORC analysis will become a fruitful area of future FORC research.

Acknowledgments

We thank Ayako Katayama for her invaluable practical assistance to this work. This work was supported financially by the National Institute of Advanced Industrial Science and Technology, Ministry of Economy, Trade and Industry, Japan (APR, HO, DH, XZ, RJH, ARM, PXH, and TS), the Australian Research Council through grant DP160100805 (APR, DH, RJH, ARM, and PXH), and by the European Research Council under the European Union's Seventh Framework

Programme (FP/2007-2013)/ERC grant agreement number 320750 (RJH). The authors thank Prof. Liao Chang for providing the magnetofossil-rich PETM sample for Figure 13. The software and data used in this paper are available from the author on request and from the FORCulator website (https:// wserv4.esc.cam.ac.uk/nanopaleomag/? page_id = 1125).

\section{References}

Bishop, C. (2006). Pattern Recognition and Machine Learning. New York: Springer.

Brownlee, S. J., Feinberg, J. M., Kasama, T., Harrison, R. J., Scott, G. R., \& Renne, P. R. (2011). Magnetic properties of ilmenite-hematite single crystals from the Ecstall pluton near Prince Rupert, British Columbia. Geochemistry, Geophysics, Geosystems, 12(9), Q07Z29. https://doi.org/10.1029/2011GC003622

Carvallo, C., \& Muxworthy, A. R. (2006). Low-temperature first-order reversal curve (FORC) diagrams for synthetic and natural samples. Geochemistry, Geophysics, Geosystems, 7(9), Q09003. https://doi.org/10.1029/2006GC001299

Carvallo, C., Muxworthy, A. R. \& Dunlop, D. J. (2006). First-order reversal curve (FORC) diagrams of magnetic mixtures: Micromagnetic models and measurements. Physics of the Earth and Planetary Interiors, 154(3-4), 308-322. https://doi.org/10.1016/j.pepi.2005.06.017

Carvallo, C., Muxworthy, A. R., Dunlop, D. J., \& Williams, W. (2003). Micromagnetic modeling of first-order reversal curve (FORC) diagrams for single-domain and pseudo-single-domain magnetite. Earth and Planetary Science Letters,

213(3-4). 375-390. https://doi.org/10.1016/S0012-821X(03)00320-0

Chang, L., Harrison, R. J., Zeng, F., Berndt, T. A., Roberts, A. P., Heslop, D., \& Zhao, X. (2018). Coupled microbial bloom and oxygenation decline recorded by magnetofossils during the Palaeocene-Eocene Thermal Maximum. Nature Communications, 9, 4007. https://doi. org/10.1038/s41467-018-06472-y

Church, N. S., Fabian, K., \& McEnroe, S. A. (2016). Nonlinear Preisach maps: Detecting and characterizing separate remanent magnetic fractions in complex natural samples. Journal of Geophysical Research - Solid Earth, 121(12), 8373-8395. https://doi.org/10.1002/ 2016JB013465

Day, R., Fuller, M., \& Schmidt, V. A. (1977). Hysteresis properties of titanomagnetites: Grain-size and compositional dependence. Physics of the Earth and Planetary Interiors, 13(4), 260-267. https://doi.org/10.1016/0031-9201(77)90108-X

Dumas, R., Li, C., Roshchin, I., Schuller, I., \& Liu, K. (2007). Magnetic fingerprints of sub- 100nm Fe dots. Physical Review B, 75(13), 1-5. Egli, R. (2006). Theoretical aspects of dipolar interactions and their appearance in first-order reversal curves of thermally activated singledomain particles. Journal of Geophysical Research - Solid Earth, 111(B12), B12S17. https://doi.org/10.1029/2006JB004567 
Egli, R. (2013). VARIFORC: An optimized protocol for calculating non-regular first-order reversal curve (FORC) diagrams. Global and Planetary Change, 110(Part C), 302-320. https://doi.org/10.1016/j.gloplacha.2013.08.003

Egli, R., Chen, A. P., Winklhofer, M., Kodama, K. P., \& Horng, C.-S. (2010). Detection of noninteracting single domain particles using firstorder reversal curve diagrams. Geochemistry, Geophysics, Geosystems, 11(1), Q01Z11. https://doi.org/10.1029/2009GC002916

Egli, R., \& Winklhofer, M. (2014). Recent developments on processing and interpretation aspects of first-order reversal curves (FORC). Proceedings of the Kazan University, 156(1), 14-53.

Fabian, K. (2003). Some additional parameters to estimate domain state from isothermal remanent magnetization. Earth and Planetary Science Letters, 213(3-4), 337-345. https://doi.org/10.1016/S0012-821X(03)00329-7

Fabian, K., \& Shcherbakov, V. P. (2004). Domain state stabilization by iterated thermal magnetization processes. Geophysical Journal International, 159(2), 486-494. https://doi.org/10.1111/j.1365-246X.2004.02394.x

Fabian, K., \& von Dobeneck, T. (1997). Isothermal magnetization of samples with stable Preisach function: A survey of hysteresis, remanence, and rock magnetic parameters. Journal of Geophysical Research - Solid Earth, 102(B8), 17,659-17,677. https://doi.org/10.1029/ 97jb01051

Harrison, R. J., Church, N., \& Fabian, K. (2017). Why do hematite FORCs look weird? . AGU Fall Meeting, New Orleans: Abstract.

Harrison, R. J., Dunin-Borkowski, R. E., \& Putnis, A. (2002). Direct imaging of nanoscale magnetic interactions in minerals. Proceedings of the National Academy of Sciences of the United States of America, 99(26), 16,556-16,561. https://doi.org/10.1073/pnas.262514499

Harrison, R. J., \& Feinberg, J. M. (2008). FORCinel: An improved algorithm for calculating first-order reversal curve distributions using locally weighted regression smoothing. Geochemistry, Geophysics, Geosystems, 9(5), Q05016. https://doi.org/10.1029/2008GC001987

Harrison, R. J., \& Lascu, I. (2014). FORCulator: A micromagnetic tool for simulating first-order reversal curve diagrams. Geochemistry, Geophysics, Geosystems, 15(12), 4671-4691. https://doi.org/10.1002/2014gc005582

Harrison, R. J., Muraszko, J., Heslop, D., Lascu, I., Muxworthy, A. R., \& Roberts, A. P. (2018). An improved algorithm for unmixing firstorder reversal curve diagrams using principal component analysis. Geochemistry, Geophysics, Geosystems, 19(5), 1595-1610. https://doi. org/10.1029/2018GC007511

Horng, C. S. (2018). Unusual magnetic properties of sedimentary pyrrhotite in methane seepage sediments: Comparison with metamorphic pyrrhotite and sedimentary greigite. Journal of Geophysical Research - Solid Earth, 123(6), 4601-4617. https://doi.org/10.1002/ 2017JB015262

Hu, P. X., Zhao, X., Roberts, A. P., Heslop, D., \& Viscarra Rossel, R. A. (2018). Magnetic domain state diagnosis in soils, loess, and marine sediments from multiple first-order reversal curve-type diagrams. Journal of Geophysical Research - Solid Earth, 123(2), 998-1017. https://doi.org/10.1002/2017jb015195

Iwaki, M. (1965). Magnetic behavior of $\alpha$ - $\mathrm{Fe}_{2} \mathrm{O}_{3}$, I. Origin of weak ferromagnetism and magnetic characteristics. Advanced Physics, 34 , 812-824. (in Japanese with English abstract)

Jacobs, I. S., \& Bean, C. P. (1955). An approach to elongated fine-particle magnets. Physical Review, 100(4), 1060-1067. https://doi.org/ 10.1103/PhysRev.100.1060

Jiang, Z. X., Q. S. Liu, M. J. Dekkers, V. Barrón, J. Torrent, and A. P. Roberts (2016). Control of Earth-like magnetic fields on the transformation of ferrihydrite to hematite and goethite, Scientific Reports, 6, 30395. https://doi.org/10.1038/srep30395

Jovane, L., Yokoyama, E., Seda, T., Burmester, R. F., Trindade, R. I. F. \& Housen, B. A. (2011). Rock magnetism of hematitic "bombs" from the Araguainha impact structure, Brazil. Geochemistry, Geophysics, Geosystems, 12(12), Q12Z34. https://doi.org/10.1029/2011GC003758

Kars, M., \& Kodama, K. (2015a). Authigenesis of magnetic minerals in gas hydrate-bearing sediments in the Nankai Trough, offshore Japan. Geochemistry, Geophysics, Geosystems, 16(3), 947-961. https://doi.org/10.1002/2014GC005614

Kars, M., \& Kodama, K. (2015b). Rock magnetic characterization of ferrimagnetic iron sulfides in gas hydrate-bearing marine sediments at Site C0008, Nankai Trough, Pacific Ocean, off-coast Japan. Earth, Planets and Space, 67(1), 118. https://doi.org/10.1186/s40623-0150287-y

Lanci, L., \& Kent, D. V. (2018). Forward modeling of thermally activated single-domain magnetic particles applied to first-order reversal curves. Journal of Geophysical Research - Solid Earth, 123(5), 3287-3300. https://doi.org/10.1002/2018JB015463

Larrasoaña, J. C., Roberts, A. P., Musgrave, R. J., Gràcia, E., Piñero, E., Vega, M. \& Martínez-Ruiz, F. (2007). Diagenetic formation of greigite and pyrrhotite in marine sedimentary systems containing gas hydrates. Earth and Planetary Science Letters, 261(3-4), 350-366. https://doi.org/10.1016/j.epsl.2007.06.032

Lascu, I., Einsle, J. F., Ball, M. R., \& Harrison, R. J. (2018). The vortex state in geologic materials: A micromagnetic perspective. Journal of Geophysical Research - Solid Earth, 123(9), 7285-7304. https://doi.org/10.1029/2018jb015909

Lascu, I., Harrison, R. J., Li, Y., Muraszko, J. R., Channell, J. E. T., Piotrowski, A. M., \& Hodell, D. A. (2015). Magnetic unmixing of firstorder reversal curve diagrams using principal component analysis. Geochemistry, Geophysics, Geosystems, 16(9), 2900-2915. https://doi. org/10.1002/2015GC005909

Liu, Q. S., Barrón, V., Torrent, J., Qin, H., \& Yu, Y. J. (2010). The magnetism of micro-sized hematite explained. Physics of the Earth and Planetary Interiors, 183(3-4), 387-397. https://doi.org/10.1016/j.pepi.2010.08.008

Ludwig, P., Egli, R., Bishop, S., Chernenko, V., Frederichs, T., Rugel, G., et al. (2013). Characterization of primary and secondary magnetite in marine sediment by combining chemical and magnetic unmixing techniques. Global and Planetary Change, 110(Part C), 321-339. https://doi.org/10.1016/j.gloplacha.2013.08.018

Martín-Hernández, F., \& Guerrero-Suárez, S. (2012). Magnetic anisotropy of hematite natural crystals: high field experiments. International Journal of Earth Sciences, 101(3), 637-647. https://doi.org/10.1007/s00531-011-0665-z

Muxworthy, A. R., Heslop, D., \& Williams, W. (2004). Influence of magnetostatic interactions on first-order-reversal-curve (FORC) diagrams: A micromagnetic approach. Geophysical Journal International, 158(3), 888-897. https://doi.org/10.1111/j.1365-246X.2004.02358.X

Muxworthy, A. R., King, J. G., \& Heslop, D. (2005). Assessing the ability of first-order reversal curve (FORC) diagrams to unravel complex magnetic signals. Journal of Geophysical Research, 110(B1), B01105. https://doi.org/10.1029/2004JB003195

Newell, A. J. (2005). A high-precision model of first-order reversal curve (FORC) functions for single-domain ferromagnets with uniaxial anisotropy. Geochemistry, Geophysics, Geosystems, 6(5), Q05010. https://doi.org/10.1029/2004GC000877

Pariona, N., Camacho-Aguilar, K. I., Ramos-González, R., Martinez, A. I., Herrera-Trejo, M., \& Baggio-Saitovitch, E. (2016). Magnetic and structural properties of ferrihydrite/hematite nanocomposites. Journal of Magnetism and Magnetic Materials, 406, 221-227. https://doi. org/10.1016/j.jmmm.2016.01.001

Pike, C., \& Fernandez, A. (1999). An investigation of magnetic reversal in submicron-scale Co dots using first order reversal curve diagrams. Journal of Applied Physics, 85(9), 6668-6676.

Pike, C. R., Roberts, A. P., Dekkers, M. J., \& Verosub, K. L. (2001). An investigation of multi-domain hysteresis mechanisms using FORC diagrams. Physics of the Earth and Planetary Interiors, 126(1-2), 11-25. https://doi.org/10.1016/S0031-9201(01)00241-2 
Pike, C. R., Roberts, A. P., \& Verosub, K. L. (1999). Characterizing interactions in fine magnetic particle systems using first order reversal curves. Journal of Applied Physics, 85(9), 6660-6667. https://doi.org/10.1063/1.370176

Pike, C. R., Roberts, A. P., \& Verosub, K. L. (2001). First-order reversal curve diagrams and thermal relaxation effects in magnetic particles. Geophysical Journal International, 145(3), 721-730. https://doi.org/10.1046/j.0956-540x.2001.01419.x

Pike, C. R., Ross, C. A., Scalettar, R. T., \& Zimanyi, G. (2005). First-order reversal curve diagram analysis of a perpendicular nickel nanopillar array. Physical Review B, 71(13), 134407.https://doi.org/10.1103/PhysRevB.71.134407

Roberts, A. P., Almeida, T. P., Church, N. S., Harrison, R. J., Heslop, D., Li, Y., et al. (2017). Resolving the origin of pseudo-single domain magnetic behavior. Journal of Geophysical Research - Solid Earth, 122(12), 9534-9558. https://doi.org/10.1002/2017JB014860

Roberts, A. P., Chang, L., Rowan, C. J., Horng, C. S., \& Florindo, F. (2011). Magnetic characteristics of sedimentary greigite (Fe3S4): An update. Reviews of Geophysics, 49(1), RG1002. https://doi.org/10.1029/2010RG000336

Roberts, A. P., Florindo, F., Larrasoaña, J. C., O’Regan, M. A., \& Zhao, X. (2010). Complex polarity pattern at the (former) Plio-Pleistocene global stratotype section at Vrica (Italy): Remagnetization by magnetic iron sulphides. Earth and Planetary Science Letters, 292(1-2), 98-111. https://doi.org/10.1016/j.epsl.2010.01.025

Roberts, A. P., Heslop, D., Zhao, X., \& Pike, C. R. (2014). Understanding fine magnetic particle systems through use of first-order reversal curve diagrams. Reviews of Geophysics, 52(4), 557-602. https://doi.org/10.1002/2014RG000462

Roberts, A. P., Hu, P. X., Harrison, R. J., Heslop, D., Muxworthy, A. R., Oda, H., et al. (2019). Domain state diagnosis in rock magnetism: Evaluation of potential alternatives to the Day diagram. Journal of Geophysical Research: Solid Earth, 124, 5286-5314 https://doi.org/ 10.1029/2018JB017049

Roberts, A. P., Liu, Q. S., Rowan, C. J., Chang, L., Carvallo, C., Torrent, J., \& Horng, C. S. (2006). Characterization of hematite ( $\alpha$-Fe $\left.{ }_{2} \mathrm{O}_{3}\right)$, goethite $(\alpha-\mathrm{FeOOH})$, greigite $\left(\mathrm{Fe}_{3} \mathrm{~S}_{4}\right)$, and pyrrhotite $\left(\mathrm{Fe}_{7} \mathrm{~S}_{8}\right)$ using first-order reversal curve diagrams. Journal of Geophysical Research, 111(B12), B12S35. https://doi.org/10.1029/2006JB004715

Roberts, A. P., Pike, C. R., \& Verosub, K. L. (2000). First order reversal curve diagrams: A new tool for characterizing the magnetic properties of natural samples. Journal of Geophysical Research - Solid Earth, 105(B12), 28,461-28,475. https://doi.org/10.1029/2000JB900326

Roberts, A. P., Tauxe, L., Heslop, D., Zhao, X., \& Jiang, Z. (2018). A critical appraisal of the "Day" diagram. Journal of Geophysical Research Solid Earth, 123(4), 2618-2644. https://doi.org/https://doi.org/10.1002/2017JB015247

Roberts, A. P., Zhao, X., Harrison, R. J., Heslop, D., Muxworthy, A. R., Rowan, C. J., et al. (2018). Signatures of reductive magnetic mineral diagenesis from unmixing of first-order reversal curves. Journal of Geophysical Research - Solid Earth, 123(6), 4500-4522. https://doi.org/ 10.1029/2018JB015706

Schabes, M. E., \& Bertram, H. N. (1988). Magnetization processes in ferromagnetic cubes. Journal of Applied Physics, 64(3), 1347-1357. https://doi.org/10.1063/1.341858

Stancu, A., Andrei, P., \& Stoleriu, L. (2006). Magnetic characterization of samples using first- and second-order reversal curve diagrams. Journal of Applied Physics, 99(8), 08D702-703. https://doi.org/10.1063/1.2172539

Valdez-Grijalva, M. A., \& Muxworthy, A. R. (2019). First-order reversal curve (FORC) diagrams of nanomagnets with cubic magnetocrystalline anisotropy: A numerical approach. Journal of Magnetism and Magnetic Materials, 471, 359-364. https://doi.org/10.1016/j. jmmm.2018.09.086

Valdez-Grijalva, M. A., Muxworthy, A. R., Williams, W., Conbhuí, P. Ó., Nagy, L., Roberts, A. P., \& Heslop, D. (2018). Magnetic vortex effects on first-order reversal curve (FORC) diagrams for greigite dispersions. Earth and Planetary Science Letters, 501, 103-111. https:// doi.org/10.1016/j.epsl.2018.08.027

Weaver, R., Roberts, A. P., \& Barker, A. J. (2002). A late diagenetic (syn-folding) magnetization carried by pyrrhotite: implications for paleomagnetic studies from magnetic iron sulphide-bearing sediments. Earth and Planetary Science Letters, 200(3), 371-386. https://doi. org/10.1016/S0012-821X(02)00652-0

Wehland, F., Stancu, A., Rochette, P., Dekkers, M. J., \& Appel, E. (2005). Experimental evaluation of magnetic interaction in pyrrhotite bearing samples. Physics of the Earth and Planetary Interiors, 153(4), 181-190. https://doi.org/10.1016/j.pepi.2005.05.006

Williams, W., \& Dunlop, D. J. (1989). Three-dimensional micromagnetic modelling of ferromagnetic domain structure. Nature, 337(6208), 634-637. https://doi.org/10.1038/337634a0

Williams, W., \& Dunlop, D. J. (1995). Simulation of magnetic hysteresis in pseudo-single-domain grains of magnetite. Journal of Geophysical Research - Solid Earth, 100(B3), 3859-3871. https://doi.org/10.1029/94jb02878

Winklhofer, M., Dumas, R. K., \& Liu, K. (2008). Identifying reversible and irreversible magnetization changes in prototype patterned media using first-and second-order reversal curves. Journal of Applied Physics, 103(7), 07C518-513. https://doi.org/10.1063/1.2837888

Yu, Y., \& Tauxe, L. (2005). On the use of magnetic transient hysteresis in paleomagnetism for granulometry. Geochemistry, Geophysics, Geosystems, 6(1), Q01H14. https://doi.org/10.1029/2004gc000839

Zhao, X., Heslop, D., \& Roberts, A. P. (2015). A protocol for variable-resolution first-order reversal curve measurements. Geochemistry, Geophysics, Geosystems, 16(5), 1364-1377. https://doi.org/10.1002/2014GC005680

Zhao, X., Roberts, A. P., Heslop, D., Paterson, G. A., Li, Y., \& Li, J. (2017). Magnetic domain state diagnosis using hysteresis reversal curves. Journal of Geophysical Research: Solid Earth, 122(7), 4767-4789. https://doi.org/10.1002/2016JB013683 